Article

\title{
Household expenditure patterns in Kuwait
}

\author{
Mohammad Alali ${ }^{1 *}$, Abdullah Aljaber ${ }^{2}$, and Adil Naseeb ${ }^{3}$ \\ 1 Kuwait Institute for Scientific Research; maali@kisr.edu.kw \\ 2 Kuwait Institute for Scientific Research; ajaber@kisr.edu.kw \\ 3 Kuwait Institute for Scientific Research; ajnaseeb@kisr.edu.kw \\ * Correspondence: maali@kisr.edu.kw.
}

Submitted: 4 December 2019; Accepted: 27 March 2020; Published: 28 March 2020

\begin{abstract}
Household expenditure is the second prominent component of GDP for Kuwait, consisting of $43 \% \mathrm{GDP}$, and it has been moderately growing for the past decade. It is essential to understand the nature of household expenditure, a fundamental macroeconomic driver with immense significance for policymaking. This paper utilises the latest Kuwait Household Expenditure Survey data to study household expenditure patterns in Kuwait. It examines and compares the variation of household expenditure patterns for nationals (Kuwaitis) and expatriate households over nine different major commodity groups. The paper investigates the patterns of household expenditure and the response of their characteristics on the level of expenditure by employing a Heckman two-step estimation method. The results suggest that different factors affect the probability of consuming a commodity and the level of expenditure between the two household groups. Kuwaiti's expenditure is more responsive to food, housing, communication, and recreation commodities and less responsive to clothing, health, transportation and restaurants than expatriates. In general, there is a significant variation of expenditure patterns across all commodities between the two household groups.
\end{abstract}

Keywords: Household Expenditure, Expenditure Elasticities, Expenditure Patterns.

JEL codes: D11, D12, D14.

\section{Introduction}

Demand-led growth studies affirm that household consumption expenditure is a principal macroeconomic driving force. Almost all economic activities are affected directly (demand-side) or indirectly (supply-side) by the level of private household expenditure, such that obvious impacts manifest in increasing or decreasing industrial production and employment levels (Tian et al., 2016; Setterfield and Kim, 2017). The importance on such phenomena has attracted the attention of several researchers investigating the expenditure patterns of households from a microeconomic perspective to provide policymakers with insights of the impacts of changes in prices, incomes and preferences on future household trends (Yusof and Duasa, 2010; Dybczak, Tóth and Voňka, 2014).

In Kuwait, household expenditure is the second-highest component of GDP after oil exports, consisting of $43 \% \mathrm{GDP}$, and it has been moderately growing for the past decade (CBK, 2016). Therefore, understanding and analysing the multifaceted aspects that determine the consumption decision and the patterns of household spending is crucial for welfare analysis and government subsidies plans in Kuwait. Despite its importance, only one previous attempt has been made to analyse household expenditure in Kuwait, by Burney and Al-Mutairi (1993). Their study applied three different functional forms of Engel curves with only two variables; household size and total expenditure, using data from the 1986/1987 household budget survey to examine the differences in expenditure patterns between Kuwaitis and expatriates irrespective of calculating their elasticities. 
Regardless of the obvious importance of such investigation, there have been no further attempts to expand on this topic, mainly due to microdata limitations. This paper uses a recent and more detailed dataset on household expenditure and demographic characteristics to estimate expenditure elasticities for the main categories of commodities within the economy. The paper applies a two-step Heckman procedure to correct for censoring bias contained within the data, an issue that was not addressed by Burney and Al-Mutairi (1993). The censored bias is contained within most household expenditure surveys (the zero expenditure observations for a commodity). Failure to correct this problem leads to biased and inconsistent results, as indicated by Heien and Wessells (1990), YeongSheng et al. (2009) and Zhang and Goddard (2010).

Additionally, this paper examines household expenditure patterns in Kuwait between expatriate and Kuwaiti households via estimating expenditure elasticities. This is particularly interesting due to the structure of the Kuwaiti population, in common with other GCC states, in having a very high expatriate population and economic regulations that discriminate between nationals and nonnationals regarding the prices of commodities and services; and the substantial differences in terms of income levels, preferences, and social lifestyles between the two groups.

The remainder of this paper proceeds as follows. Section 2 discusses briefly the theoretical foundation. Section 3 describes the methodological choices of functional form and the estimation procedure employed. Section 4 introduces the data, provides the sample data descriptions of variables used and summary statistics. Section 5 presents the empirical results and discusses the findings. Section 6 provides the conclusion and recommendations for further study and policy.

\section{Theoretical Foundations}

Examining the existing literature on household expenditure patterns offers countless methods for its modelling; one of the most effective methods among others is the utilization of Engel curves. Ernst Engel studied the relationship between income and the expenditure on specific goods and services, a relationship known as the Engel curve. The practice of Engel curves was popularised in modelling household expenditure by Houthakker (1957), who studied the use of the curves for determining the income elasticities of demand for households and compared income elasticities for food, housing, clothing and miscellaneous items for thirty different countries. Since then the applications of Engel curves have been appropriated in understanding how households allocate their income between different commodities. These curves have several significant advantages than any other method. They can define the demand for a commodity by describing the relationship between income and the expenditure, regardless of expressing a utility function (Barnett and Serletis, 2008). Often, Engel curves are expressed as expenditure shares (the total expenditure assigned to a specific commodity from total income), rather than relating total expenditure directly to total income.

Moreover, this association describes how the ratio of total income spent on a commodity differs with income. Besides, another advantage of using Engle curves for economists studying household behaviour is the straightforward computation of income elasticities, which represent the change in quantity demanded given a change in income. Obtaining income elasticities provides an indicator of how different households categorize their behaviour for consuming a specific good. For example, a good with an income elasticity above one is classified as a luxury good while a necessity good is defined to have an elasticity of less than one. Thus, income elasticities can be used as an indicator of the impact of a change in income on future expenditures patterns (Chandran et al., 2015).

Additionally, Engel curves can be extended to include demographic factors that influence the expenditure behaviour of households. This extension has an essential role in measuring the changes in quantity demanded a commodity given a change in household income while taking into consideration the distinctive characteristics of households, to better understand their expenditure behaviour and patterns. There are many functional forms of Engel curves, and collectively they are one of the most applied methods in empirical analyses of household expenditure patterns. Hence, these curves have been a favourite instrument for studying welfare analysis of different households 
and the response of different factors like income, prices and other demographic factors on expenditure. (Deaton and Muellbauer, 1980; Michelini and Chatterjee, 1997; Durham and Eales, 2010).

This paper differs from the existing literature as it reveals a range of demographic factors that affect household expenditure behaviour, represented by the social status and lifestyles of households. Also, this paper employs a well-known Engel curve suggested by Working (1943) and Leser (1963), to estimate expenditure elasticities. The Working-Leser Engel curve was estimated according to the recognised method for accounting for censoring data issues, which is the Heckman (1979) two-step regression method.

\section{Methodology}

To analyse household expenditure patterns and determine the factors that influence their expenditure behaviour, an appropriate functional form must be identified. The functional form must satisfy the restrictions of the consumer demand theory. The Working-Leser Engel curve proposed by Working (1943) and Leser (1963) is a widely used functional form in recent studies that are derived from a utility maximization problem and consistent with the general demand theory. This model establishes a functional relationship between household expenditure on a commodity as a proportion of total income with the assumption of a linear budget function (Mok et al., 2011 and Lokshin, 2016; De Agostini, 2014).

However, in most empirical research on household expenditure, the usage of total expenditure as a proxy for income is preferable, as income level data commonly suffer from numerous measurement errors in most household budget surveys, especially in economies with no or minimal taxes, wherein households generally tend to miss-specify their total income. Therefore, throughout this paper income and expenditure indicate the same variable. Hence, the basic Working-Leser model will have the following form:

$$
s_{i}=\alpha_{i}+\beta_{i} E X P+\varepsilon_{i}
$$

where $s_{i}$ indicates the expenditure share on commodity, EXP is the log of total expenditure, and $\varepsilon_{i}$ is an error term distributed normally.

According to Deaton and Muellbauer (1980), the use of OLS regression will automatically satisfy the adding up restriction that states $\sum_{i=1}^{n} \quad \alpha_{i}=1$ and $\sum_{i=1}^{n} \quad \beta_{i}=0$. Besides, the above model reflects that the estimated $\beta_{i}$ coefficient measures the effect of changes in total expenditure on the expenditure share of a commodity. This coefficient specifies whether a commodity is a luxury or a necessity. A commodity is a luxury good if $\beta_{i}>0$ and a necessity good if $\beta_{i}<0$. However, since there is a variation between the household's expenditure shares, to identify goods as luxury or necessity on the average household, the expenditure elasticities are calculated at the mean expenditure shares. Hence, the expenditure elasticities for this model can be expressed as noted by Chandran et al. (2015):

$$
e=1+\left(\frac{\beta_{i}}{s_{i}}\right)
$$

where the expenditure elasticity for the average household is a luxury good if $\mathrm{e}>0$ and a necessity good if $\mathrm{e}<0$.

In line with the literature, a vector of dummy household characteristics was introduced linearly to equation (1), to capture the different effects of demographic variables on household preferences towards consumption. The demographic variables that are extended to the basic Working-Leser model equation (1) are the Head of Household $(\mathrm{HoH})$, age, sex, marital status, employment status and level of education. Besides, the size and the number of children in the household are introduced. Hence, the final extended model that will be utilised throughout this research is expressed in the same way as in equation (2) but with the extension of the variable $h_{k}$, which indicates different demographic variables. 
The final extended model is shown in equation (3), which is used to separately estimate the factors influencing the consumption behaviour of Kuwaiti and expatriate households.

$$
s_{i}=\alpha_{i}+\beta_{i} \log E X P+\sum_{k} \quad \delta_{i k} h_{k}+\varepsilon_{i}
$$

This paper applies the Heckman two-step technique to address the problem of zero expenditure observations and provide more robust and consistent estimates than other methods. The estimation of the Heckman two-step method is obtained by first estimating a Probit model using a maximum likelihood estimation procedure including all zero and non-zero observations, to capture the factors that influence the probability of a household consuming a commodity. From this regression, a vector of the inverse Mills ratio (MILLS) is calculated and used in the second step as an additional independent variable (MILLS indicates the predicted probability of a household consuming a commodity). The second step is estimated for the commodity of interest by a standard OLS regression on only the non-zero observation within the sample. Mathematically, the estimation procedure will proceed as described below, following the method outlined by Bushway, Johnson, and Slocum (2007).

Equation (4) represents the first step of the Heckman procedure, which is the probability of a household consuming a commodity.

$$
y_{i}=\beta_{i} \log E X P+\sum_{k} \delta_{i k} h_{k}+\varepsilon_{i}
$$

where $y_{i}$ is one if a household consumes the $i^{\text {th }}$ commodity and zero otherwise. The independent variables that influence the expenditure decision are included ${ }^{1}$, and $\varepsilon_{i}$ is an error term distributed normally. For further illustration, equation (4) can also be expressed as:

$$
\operatorname{Pr} \operatorname{Pr}\left[y_{i}=1\right]=\Phi\left(X \gamma_{i}\right)
$$

where $\mathrm{X}$ is a vector of the regressors used above, $\gamma_{i}$ is the coefficients to be estimated for the regressors and $\Phi$ is the normal cumulative distribution function. The estimates from the Probit model are then used to calculate the MILLS for each household by dividing the normal density function $(\phi)$ by $1-\Phi$.

The second step can be shown as:

$$
M I L L S=\frac{\phi\left(X \gamma_{i}\right)}{1-\Phi\left(X \gamma_{i}\right)}
$$

$$
s_{i}=\alpha_{i}+\beta_{i} \log E X P+\sum_{k} \delta_{i k} h_{k}+\gamma_{i} M I L L S+\varepsilon_{i}
$$

Equation (6) is estimated for observations employed for households that did not report zero expenditures. This model is considered as the final model and was employed for the two types of households.

\section{Data and Descriptive Statistics}

This study utilises the most recent Kuwait Household Expenditure Survey (2013) provided by the Central Statistical Bureau. The survey consists of 2,961 households, comprising 1,226 Kuwaiti and 1,735 expatriate households, respectively. The survey collects information on the total expenditure of each household on a comprehensive list of disaggregated commodities, in addition to the characteristics of household members. For this study, the commodities have been aggregated into nine commodities based on similar characteristics. Households, which did not report any expenditure, were excluded from the analysis alongside with the outliers, verified from visual and statistical examinations. As a result, the final sample consists of 1,090 Kuwaiti and 1,616 expatriate households'

\footnotetext{
1 The independent variables included are the same as expressed in equation (3): log of total expenditure, the HoH age, sex marital status, employment status and level of education. Also, the size of the household and the number of children are included.
} 
expenditure on the following commodity groups, which are classified by the United Nations as a classification of individual consumption by purpose:

- food,

- clothing,

- housing,

- health,

- transport,

- communication,

- recreation and culture,

- hotels and restaurants,

- miscellaneous goods and services.

Table 1. Variables Representation

\begin{tabular}{|c|c|}
\hline Variables & Description \\
\hline & Dependent Variables $\left(S_{i}\right)$ \\
\hline Food & Expenditure share on Food \\
\hline Clothing & Expenditure share on Clothing and footwear \\
\hline Housing & $\begin{array}{l}\text { Expenditure share on Housing (includes water, electricity, gas, } \\
\text { furnishing and household equipment) }\end{array}$ \\
\hline Health & Expenditure share on Health services \\
\hline Trans. & Expenditure share on Transport \\
\hline Comm. & Expenditure share on Communication \\
\hline Rec. & Expenditure share on Recreation and culture \\
\hline Rest. & Expenditure share on Restaurants and hotels \\
\hline Misc. & Expenditure share on Miscellaneous goods and services \\
\hline \multirow{3}{*}{ EXP } & Independent Variables $\left(\mathrm{X}_{\mathrm{i}}\right)$ \\
\hline & Natural Log of Total Expenditure per quarter \\
\hline & Demographic Variables \\
\hline Size & Number of members within the household \\
\hline Age & Age of $\mathrm{HoH}$ \\
\hline EMP & Employment Status of $\mathrm{HoH}$ \\
\hline Marital & Marital Status of $\mathrm{HoH}$ \\
\hline Sex & Sex of $\mathrm{HoH}$ \\
\hline EDU & The educational level of $\mathrm{HoH}$ \\
\hline Children & Number of members under the age of 18 \\
\hline
\end{tabular}

Source: Authors

Table 1 provides descriptions of the main dependent $(\mathrm{Si})$ and independent $(\mathrm{Xi})$ variables gathered from the survey. In line with the literature, the dependent variables are expressed as expenditure shares. To achieve the objectives of this study, operational definitions and parameters were used and given in Table 1. Most of the demographic variables included in our model are presented as categorical variables aside from the number of members under the age of 18 (Children). The purpose of using categorical variables is to capture the different effects of different types of households. Therefore, in the estimation process, one group from each categorical variable will be left out as a reference group, to avoid collinearity. 
The descriptive statistics of total expenditure, expenditure shares and demographic characteristics of the sample in the study of the two different subgroups are presented in Table 2, with mean and standard deviation (SD). The table provides an insight into the differences in household expenditure patterns, reflected by the average expenditure shares of nine commodities for both Kuwaitis and expatriates. The data confirms the disparity between the total expenditure (which is a proxy for total income) of Kuwaiti and expatriate households.

Table 2. Summary Statistics for Total Expenditure, Expenditure Shares, and Household Characteristics

\begin{tabular}{|c|c|c|c|c|}
\hline \multirow{2}{*}{ Variables } & \multicolumn{2}{|c|}{ Kuwaiti } & \multicolumn{2}{|c|}{ Expatriate } \\
\hline & Mean & SD & Mean & SD \\
\hline EXP & 7323 & 4281 & 3796 & 1624 \\
\hline Food & 0.08 & 0.13 & 0.09 & 0.14 \\
\hline Clothing & 0.10 & 0.14 & 0.09 & 0.11 \\
\hline Housing & 0.17 & 0.19 & 0.21 & 0.22 \\
\hline Health & 0.01 & 0.02 & 0.01 & 0.02 \\
\hline Trans. & 0.08 & 0.16 & 0.05 & 0.10 \\
\hline Comm. & 0.01 & 0.04 & 0.03 & 0.06 \\
\hline Rec. & 0.03 & 0.05 & 0.03 & 0.06 \\
\hline Rest. & 0.02 & 0.04 & 0.01 & 0.05 \\
\hline Misc. & 0.44 & 0.22 & 0.43 & 0.21 \\
\hline \multicolumn{5}{|l|}{ Size base (1-2) } \\
\hline $3-4$ & 0.19 & 0.39 & 0.38 & 0.39 \\
\hline $5-7$ & 0.24 & 0.43 & 0.12 & 0.43 \\
\hline$\geq 8$ & 0.47 & 0.50 & 0.08 & 0.50 \\
\hline \multicolumn{5}{|l|}{ Age base (20-39) } \\
\hline $40-47$ & 0.21 & 0.41 & 0.29 & 0.41 \\
\hline $48-56$ & 0.23 & 0.42 & 0.21 & 0.42 \\
\hline$\geq 57$ & 0.35 & 0.48 & 0.18 & 0.48 \\
\hline \multicolumn{5}{|l|}{ EDU base (low) } \\
\hline Moderate & 0.37 & 0.48 & 0.30 & 0.48 \\
\hline High & 0.26 & 0.44 & 0.43 & 0.44 \\
\hline \multicolumn{5}{|l|}{ EMP (base Employed) } \\
\hline Others & 0.48 & 0.50 & 0.03 & 0.50 \\
\hline \multicolumn{5}{|l|}{ Sex (base male) } \\
\hline Female & 0.15 & 0.36 & 0.04 & 0.36 \\
\hline \multicolumn{5}{|l|}{ Marital base (single) } \\
\hline Married & 0.82 & 0.39 & 0.95 & 0.39 \\
\hline Children & 2.57 & 2.39 & 1.52 & 2.39 \\
\hline Number of Observations & \multicolumn{2}{|c|}{1,090} & \multicolumn{2}{|c|}{1,616} \\
\hline
\end{tabular}

Source: Authors Calculation

The average Kuwaiti household spends 2,441 KD per month on all commodities, while an expatriate household spends only 1,265 KD. Aside from this elementary divergence, on average, the expenditure shares for almost all commodities are different between the two groups, except for health and recreation and culture. Expatriates spend relatively more on food, housing, and communication, with around $9 \%, 21 \%$, and 3\% of their total expenditure, respectively; Kuwaiti households spend more on clothing, restaurants and hotels, transportation and miscellaneous goods and services, with $10 \%$, $2 \%, 8 \%$, and $43 \%$ of their budget share, respectively. For both groups, miscellaneous goods and services consume the lion's share of their expenditure, which is likely attributable to the large and diverse range of disaggregated products and services that comprise this commodity group, particularly large expenditure items like financial services (loans and mortgages).

Since this study uses micro-level household expenditure data, the threat of households reporting missing and zero expenditure on commodities exists. This study assumes missing observations are 
considered as a non-consumption decision made by the household; hence, missing observations were converted to zeros. There are many explanations for observing zero expenditure or missing observations within the data. Table 3 displays the percentage of households with zero expenditure shares across all commodities. Most of the households reported zero expenditure on health since most public health services are free of charge. Restaurants and hotels were the second-largest commodity group after health. On the other hand, the least reported commodity was miscellaneous goods and services, which might indicate the importance of this commodity to households.

Table 3. Percentage of Households with Zero Expenditure Across the Whole Sample

\begin{tabular}{lcc}
\hline \multicolumn{1}{c}{ Commodity Groups } & Kuwaiti & Expatriate \\
\hline Food and non-alcoholic beverages & 27 & 26 \\
Clothing and footwear & 10 & 12 \\
Housing (includes water, electricity, gas & 17 & 17 \\
and other fuels) & 50 & 54 \\
Health & 21 & 25 \\
Transport & 26 & 23 \\
Communication & 28 & 30 \\
Recreation and culture & 37 & 46 \\
Restaurants and hotels & 7 & 11 \\
Miscellaneous goods and services & &
\end{tabular}

Note: Figures are in percentages

\section{Results and findings}

The research starts its empirical analysis by estimating the first-step equation (4) of the Heckman procedure. Table 4.1-4.2 presents the marginal effects for the Probit estimates for Kuwait and expatriate households. The results generally indicate that there is a significant variation between the two groups, which confirms the different impacts of household characteristics on consumption. However, there are some similarities between the two groups. The results for both households show that total expenditure has a positive and statistically significant impact on the probability of consumption across all commodities. They also show that households are less likely to spend on housing, restaurants, as they get older, and the size of the family increases. Besides, the level of education tends to decrease the probability of consuming communication goods and services. Likewise, the probability of consuming clothing products rises among female $\mathrm{HoH}$ compared with males for both groups.

On the other hand, there are many differences between the two households; some demographic variables affect the probability of consumption for one group but not for the other. The Kuwaiti household's marginal effects on consuming food are significant with total expenditure only. Total expenditure as well as household size, sex of $\mathrm{HoH}$ and the number of children significantly affect expatriate's decision of consuming food. Also, the decision on spending on clothing for Kuwaitis was significantly related to the educational level and the sex of $\mathrm{HoH}$, whereas for expatriates it was significantly related to employment status, marital status and the presence of children. It was also observed that the number of children for Kuwaitis influences the probability of spending on housing, while for expatriates the probability of spending on housing was affected by only the sex and marital status of $\mathrm{HoH}$. 
Table 4.1. Marginal Effects for Probits Estimates for Kuwaiti Households

\begin{tabular}{|c|c|c|c|c|c|c|c|c|c|}
\hline VARIABLES & $\begin{array}{c}(1) \\
\text { Food }\end{array}$ & $\begin{array}{c}(2) \\
\text { Clothing } \\
\end{array}$ & $\begin{array}{c}(3) \\
\text { Housing } \\
\end{array}$ & $\begin{array}{c}(4) \\
\text { Health }\end{array}$ & $\begin{array}{c}(5) \\
\text { Trans. }\end{array}$ & $\begin{array}{c}(6) \\
\text { Comm. }\end{array}$ & $\begin{array}{l}(7) \\
\text { Rec. }\end{array}$ & $\begin{array}{l}(8) \\
\text { Res. }\end{array}$ & $\begin{array}{c}(9) \\
\text { Misc. }\end{array}$ \\
\hline EXP & $\begin{array}{l}0.103^{* * *} \\
(0.00918)\end{array}$ & $\begin{array}{l}0.0671^{* * *} \\
(0.00566)\end{array}$ & $\begin{array}{l}0.106^{* * *} \\
(0.00608)\end{array}$ & $\begin{array}{l}0.190^{* * *} \\
(0.0115)\end{array}$ & $\begin{array}{l}0.122^{* * *} \\
(0.00768)\end{array}$ & $\begin{array}{c}0.144^{* * *} \\
(0.00849)\end{array}$ & $\begin{array}{l}0.148^{* * *} \\
(0.00819)\end{array}$ & $\begin{array}{l}0.171^{* * *} \\
(0.0106)\end{array}$ & $\begin{array}{l}0.0490^{* * *} \\
(0.00456)\end{array}$ \\
\hline \multicolumn{10}{|l|}{$\begin{array}{l}\text { Size (base } 1- \\
2 \text { ) }\end{array}$} \\
\hline $3-5$ & $\begin{array}{l}-0.0654 \\
(0.0517)\end{array}$ & $\begin{array}{l}0.0456^{*} \\
(0.0272)\end{array}$ & $\begin{array}{l}0.00799 \\
(0.0285)\end{array}$ & $\begin{array}{c}0.0119 \\
(0.0524)\end{array}$ & $\begin{array}{l}-0.00819 \\
(0.0405)\end{array}$ & $\begin{array}{l}-0.0309 \\
(0.0376)\end{array}$ & $\begin{array}{l}0.00924 \\
(0.0390)\end{array}$ & $\begin{array}{l}-0.0377 \\
(0.0469)\end{array}$ & $\begin{array}{c}-0.00469 \\
(0.0142)\end{array}$ \\
\hline $6-7$ & $\begin{array}{c}0.0157 \\
(0.0504)\end{array}$ & $\begin{array}{c}0.0418 \\
(0.0298)\end{array}$ & $\begin{array}{l}-0.0571^{*} \\
(0.0313)\end{array}$ & $\begin{array}{l}-0.0168 \\
(0.0550)\end{array}$ & $\begin{array}{l}-0.0385 \\
(0.0415)\end{array}$ & $\begin{array}{l}-0.0643 \\
(0.0393)\end{array}$ & $\begin{array}{l}-0.0435 \\
(0.0420)\end{array}$ & $\begin{array}{l}-0.0836^{*} \\
(0.0477)\end{array}$ & $\begin{array}{l}0.00257 \\
(0.0151)\end{array}$ \\
\hline $8>$ & $\begin{array}{l}-0.0281 \\
(0.0546)\end{array}$ & $\begin{array}{c}0.0477 \\
(0.0310)\end{array}$ & $\begin{array}{c}-0.0703^{* *} \\
(0.0349)\end{array}$ & $\begin{array}{l}-0.0358 \\
(0.0578)\end{array}$ & $\begin{array}{c}0.000836 \\
(0.0440)\end{array}$ & $\begin{array}{l}-0.0533 \\
(0.0433)\end{array}$ & $\begin{array}{l}-0.0732 \\
(0.0479)\end{array}$ & $\begin{array}{l}-0.0355 \\
(0.0514)\end{array}$ & $\begin{array}{l}-0.0147 \\
(0.0174)\end{array}$ \\
\hline \multicolumn{10}{|l|}{$\begin{array}{c}\text { Age (base } 20 \\
-39)\end{array}$} \\
\hline $40-47$ & $\begin{array}{l}-0.0410 \\
(0.0394)\end{array}$ & $\begin{array}{c}-0.0511^{* * *} \\
(0.0179)\end{array}$ & $\begin{array}{c}0.0120 \\
(0.0325)\end{array}$ & $\begin{array}{c}0.0273 \\
(0.0413)\end{array}$ & $\begin{array}{c}0.0289 \\
(0.0304)\end{array}$ & $\begin{array}{l}-0.0173 \\
(0.0348)\end{array}$ & $\begin{array}{c}0.0486 \\
(0.0344)\end{array}$ & $\begin{array}{l}-0.0360 \\
(0.0377)\end{array}$ & $\begin{array}{c}-0.00216 \\
(0.0165)\end{array}$ \\
\hline $48-56$ & $\begin{array}{l}0.00424 \\
(0.0409)\end{array}$ & $\begin{array}{c}-0.0553^{* * *} \\
(0.0172)\end{array}$ & $\begin{array}{c}0.0364 \\
(0.0341)\end{array}$ & $\begin{array}{l}-0.0423 \\
(0.0461)\end{array}$ & $\begin{array}{l}-0.0284 \\
(0.0348)\end{array}$ & $\begin{array}{l}-0.0649^{*} \\
(0.0383)\end{array}$ & $\begin{array}{c}0.0194 \\
(0.0406)\end{array}$ & $\begin{array}{l}-0.0898^{* *} \\
(0.0430)\end{array}$ & $\begin{array}{c}-0.00330 \\
(0.0162)\end{array}$ \\
\hline $57>$ & $\begin{array}{c}-0.00338 \\
(0.0450)\end{array}$ & $\begin{array}{c}-0.0483^{* * *} \\
(0.0176)\end{array}$ & $\begin{array}{c}0.0334 \\
(0.0354)\end{array}$ & $\begin{array}{l}-0.0631 \\
(0.0489)\end{array}$ & $\begin{array}{l}-0.0191 \\
(0.0381)\end{array}$ & $\begin{array}{l}-0.0620 \\
(0.0395)\end{array}$ & $\begin{array}{l}-0.0375 \\
(0.0443)\end{array}$ & $\begin{array}{l}-0.0513 \\
(0.0454)\end{array}$ & $\begin{array}{c}-0.00174 \\
(0.0185)\end{array}$ \\
\hline $\begin{array}{c}\text { EDU (base } \\
\text { low) }\end{array}$ & & & & & & & & & \\
\hline Moderate & $\begin{array}{l}-0.0145 \\
(0.0305)\end{array}$ & $\begin{array}{c}-0.00909 \\
(0.0140)\end{array}$ & $\begin{array}{c}0.000387 \\
(0.0216)\end{array}$ & $\begin{array}{l}-0.0262 \\
(0.0325)\end{array}$ & $\begin{array}{l}-0.0221 \\
(0.0254)\end{array}$ & $\begin{array}{l}-0.0255 \\
(0.0266)\end{array}$ & $\begin{array}{c}0.0415 \\
(0.0274)\end{array}$ & $\begin{array}{l}-0.0280 \\
(0.0314)\end{array}$ & $\begin{array}{l}0.00399 \\
(0.0124)\end{array}$ \\
\hline High & $\begin{array}{l}-0.0318 \\
(0.0351)\end{array}$ & $\begin{array}{c}-0.0526^{* * *} \\
(0.0185)\end{array}$ & $\begin{array}{l}0.00829 \\
(0.0254)\end{array}$ & $\begin{array}{l}-0.0573 \\
(0.0373)\end{array}$ & $\begin{array}{l}-0.00353 \\
(0.0274)\end{array}$ & $\begin{array}{l}-0.0591^{*} \\
(0.0320)\end{array}$ & $\begin{array}{l}-0.0383 \\
(0.0342)\end{array}$ & $\begin{array}{l}-0.0469 \\
(0.0366)\end{array}$ & $\begin{array}{l}0.00754 \\
(0.0139)\end{array}$ \\
\hline \multicolumn{10}{|l|}{$\begin{array}{l}\text { EMP (base } \\
\text { Employed) }\end{array}$} \\
\hline Others & $\begin{array}{l}-0.0461 \\
(0.0343)\end{array}$ & $\begin{array}{l}0.00339 \\
(0.0148)\end{array}$ & $\begin{array}{c}0.0133 \\
(0.0236)\end{array}$ & $\begin{array}{c}0.0943^{* * *} \\
(0.0360)\end{array}$ & $\begin{array}{c}0.0195 \\
(0.0289)\end{array}$ & $\begin{array}{l}0.0517^{*} \\
(0.0300)\end{array}$ & $\begin{array}{c}0.0425 \\
(0.0311)\end{array}$ & $\begin{array}{c}0.0207 \\
(0.0349)\end{array}$ & $\begin{array}{l}0.00814 \\
(0.0143)\end{array}$ \\
\hline \multicolumn{10}{|l|}{$\begin{array}{l}\text { Sex (base } \\
\text { male) }\end{array}$} \\
\hline Female & $\begin{array}{c}0.0144 \\
(0.0537)\end{array}$ & $\begin{array}{l}0.0395^{* *} \\
(0.0189)\end{array}$ & $\begin{array}{l}-0.0257 \\
(0.0501)\end{array}$ & $\begin{array}{c}0.0203 \\
(0.0588)\end{array}$ & $\begin{array}{l}-0.0582 \\
(0.0517)\end{array}$ & $\begin{array}{c}0.0117 \\
(0.0500)\end{array}$ & $\begin{array}{c}-0.00678 \\
(0.0479)\end{array}$ & $\begin{array}{l}-0.00148 \\
(0.0569)\end{array}$ & $\begin{array}{l}-0.0177 \\
(0.0227)\end{array}$ \\
\hline \multicolumn{10}{|l|}{$\begin{array}{l}\text { Marital (base } \\
\text { single) }\end{array}$} \\
\hline Others & $\begin{array}{c}0.0210 \\
(0.0543)\end{array}$ & $\begin{array}{c}0.0389 \\
(0.0302)\end{array}$ & $\begin{array}{c}-0.00963 \\
(0.0417)\end{array}$ & $\begin{array}{l}0.00812 \\
(0.0571)\end{array}$ & $\begin{array}{r}-0.00522 \\
(0.0428)\end{array}$ & $\begin{array}{c}0.0373 \\
(0.0533)\end{array}$ & $\begin{array}{l}0.0829 * \\
(0.0500)\end{array}$ & $\begin{array}{c}0.0505 \\
(0.0557)\end{array}$ & $\begin{array}{l}0.00479 \\
(0.0198)\end{array}$ \\
\hline Children & $\begin{array}{l}-0.00345 \\
(0.00698)\end{array}$ & $\begin{array}{l}-0.00413 \\
(0.00286)\end{array}$ & $\begin{array}{c}0.0128^{* *} \\
(0.00563)\end{array}$ & $\begin{array}{c}0.0100 \\
(0.00753)\end{array}$ & $\begin{array}{l}-0.00504 \\
(0.00534)\end{array}$ & $\begin{array}{c}0.00855 \\
(0.00666)\end{array}$ & $\begin{array}{l}0.0215^{* *} \\
(0.00895)\end{array}$ & $\begin{array}{c}0.00732 \\
(0.00737)\end{array}$ & $\begin{array}{c}0.00396 \\
(0.00310)\end{array}$ \\
\hline Observations & 1,090 & 1,090 & 1,090 & 1,090 & 1,090 & 1,090 & 1,090 & 1,090 & 1,090 \\
\hline
\end{tabular}


Table 4.2. Marginal Effects for Probits Estimates for Expatriate Household

\begin{tabular}{|c|c|c|c|c|c|c|c|c|c|}
\hline VARIABLES & $\begin{array}{c}(1) \\
\text { Food }\end{array}$ & $\begin{array}{c}(2) \\
\text { Clothing }\end{array}$ & $\begin{array}{c}(3) \\
\text { Housing }\end{array}$ & $\begin{array}{c}(4) \\
\text { Health }\end{array}$ & $\begin{array}{l}(5) \\
\text { Trans. }\end{array}$ & $\begin{array}{c}(6) \\
\text { Comm. }\end{array}$ & $\begin{array}{l}(7) \\
\text { Rec. }\end{array}$ & $\begin{array}{l}(8) \\
\text { Res. }\end{array}$ & $\begin{array}{l}(9) \\
\text { Misc. }\end{array}$ \\
\hline EXP & $\begin{array}{l}0.0949 * * * \\
(0.00765)\end{array}$ & $\begin{array}{l}0.0827^{* * *} \\
(0.00520)\end{array}$ & $\begin{array}{c}0.115^{* * *} \\
(0.00545)\end{array}$ & $\begin{array}{l}0.243^{* * *} \\
(0.0106)\end{array}$ & $\begin{array}{c}0.151^{* * *} \\
(0.00752)\end{array}$ & $\begin{array}{c}0.149^{* * *} \\
(0.00698)\end{array}$ & $\begin{array}{c}0.178^{* * *} \\
(0.00755)\end{array}$ & $\begin{array}{c}0.173^{* * *} \\
(0.00946)\end{array}$ & $\begin{array}{l}0.0752^{* * *} \\
(0.00479)\end{array}$ \\
\hline \multicolumn{10}{|l|}{ Size (base 1-2) } \\
\hline $3-5$ & $\begin{array}{l}0.0507^{*} \\
(0.0284)\end{array}$ & $\begin{array}{l}0.00347 \\
(0.0139)\end{array}$ & $\begin{array}{c}-0.0400^{* *} \\
(0.0198)\end{array}$ & $\begin{array}{l}-0.0118 \\
(0.0285)\end{array}$ & $\begin{array}{l}-0.0111 \\
(0.0232)\end{array}$ & $\begin{array}{r}-0.00908 \\
(0.0217)\end{array}$ & $\begin{array}{c}0.0295 \\
(0.0250)\end{array}$ & $\begin{array}{c}0.0222 \\
(0.0300)\end{array}$ & $\begin{array}{l}0.0222^{* *} \\
(0.0113)\end{array}$ \\
\hline $6-7$ & $\begin{array}{l}0.0724^{*} \\
(0.0433)\end{array}$ & $\begin{array}{l}-0.0493 \\
(0.0302)\end{array}$ & $\begin{array}{l}-0.0480 \\
(0.0332)\end{array}$ & $\begin{array}{l}-0.0493 \\
(0.0453)\end{array}$ & $\begin{array}{l}-0.0660^{*} \\
(0.0379)\end{array}$ & $\begin{array}{l}-0.0573 \\
(0.0358)\end{array}$ & $\begin{array}{c}0.0181 \\
(0.0414)\end{array}$ & $\begin{array}{c}0.0104 \\
(0.0453)\end{array}$ & $\begin{array}{l}-0.0336 \\
(0.0248)\end{array}$ \\
\hline $8>$ & $\begin{array}{l}0.0992^{*} \\
(0.0582)\end{array}$ & $\begin{array}{c}-0.0272 \\
(0.0365)\end{array}$ & $\begin{array}{c}0.0236 \\
(0.0377)\end{array}$ & $\begin{array}{c}-0.00623 \\
(0.0655)\end{array}$ & $\begin{array}{l}-0.0529 \\
(0.0585)\end{array}$ & $\begin{array}{l}-0.0922 \\
(0.0601)\end{array}$ & $\begin{array}{l}-0.0912 \\
(0.0767)\end{array}$ & $\begin{array}{c}0.0292 \\
(0.0683)\end{array}$ & $\begin{array}{l}-0.0304 \\
(0.0297)\end{array}$ \\
\hline \multicolumn{10}{|l|}{ Age (base 20 -39) } \\
\hline $40-47$ & $\begin{array}{l}-0.0253 \\
(0.0270)\end{array}$ & $\begin{array}{c}-0.0223 \\
(0.0157)\end{array}$ & $\begin{array}{c}0.0219 \\
(0.0181)\end{array}$ & $\begin{array}{c}-0.0608^{* *} \\
(0.0269)\end{array}$ & $\begin{array}{l}-0.0149 \\
(0.0237)\end{array}$ & $\begin{array}{r}-0.00123 \\
(0.0218)\end{array}$ & $\begin{array}{c}0.0142 \\
(0.0227)\end{array}$ & $\begin{array}{l}-0.0380 \\
(0.0287)\end{array}$ & $\begin{array}{c}-0.0299^{* *} \\
(0.0130)\end{array}$ \\
\hline $48-56$ & $\begin{array}{l}-0.00769 \\
(0.0296)\end{array}$ & $\begin{array}{l}-0.0156 \\
(0.0170)\end{array}$ & $\begin{array}{c}-0.0361^{*} \\
(0.0216)\end{array}$ & $\begin{array}{l}-0.0123 \\
(0.0301)\end{array}$ & $\begin{array}{c}-0.0184 \\
(0.0260)\end{array}$ & $\begin{array}{l}-0.0260 \\
(0.0239)\end{array}$ & $\begin{array}{l}-0.0149 \\
(0.0253)\end{array}$ & $\begin{array}{l}-0.116^{* * *} \\
(0.0320)\end{array}$ & $\begin{array}{l}0.00176 \\
(0.0142)\end{array}$ \\
\hline $57>$ & $\begin{array}{c}0.0225 \\
(0.0314)\end{array}$ & $\begin{array}{c}0.0133 \\
(0.0158)\end{array}$ & $\begin{array}{l}-0.0199 \\
(0.0245)\end{array}$ & $\begin{array}{l}-0.0238 \\
(0.0335)\end{array}$ & $\begin{array}{c}-0.00815 \\
(0.0264)\end{array}$ & $\begin{array}{c}0.0325 \\
(0.0247)\end{array}$ & $\begin{array}{l}-0.0167 \\
(0.0283)\end{array}$ & $\begin{array}{c}-0.0922^{* * *} \\
(0.0350)\end{array}$ & $\begin{array}{l}-0.0122 \\
(0.0147)\end{array}$ \\
\hline \multicolumn{10}{|l|}{ EDU (base low) } \\
\hline Moderate & $\begin{array}{l}0.00401 \\
(0.0277)\end{array}$ & $\begin{array}{c}-0.0173 \\
(0.0152)\end{array}$ & $\begin{array}{l}-0.0217 \\
(0.0201)\end{array}$ & $\begin{array}{l}-0.0347 \\
(0.0289)\end{array}$ & $\begin{array}{c}0.0101 \\
(0.0246)\end{array}$ & $\begin{array}{c}-0.0426^{* *} \\
(0.0217)\end{array}$ & $\begin{array}{c}0.0217 \\
(0.0245)\end{array}$ & $\begin{array}{c}0.0832 * * * \\
(0.0302)\end{array}$ & $\begin{array}{l}0.00672 \\
(0.0118)\end{array}$ \\
\hline High & $\begin{array}{l}0.00509 \\
(0.0269)\end{array}$ & $\begin{array}{r}-0.00891 \\
(0.0146)\end{array}$ & $\begin{array}{l}-0.0204 \\
(0.0192)\end{array}$ & $\begin{array}{l}0.00134 \\
(0.0280)\end{array}$ & $\begin{array}{l}0.00475 \\
(0.0239)\end{array}$ & $\begin{array}{c}-0.0583^{* * *} \\
(0.0215)\end{array}$ & $\begin{array}{c}0.0710^{* * * *} \\
(0.0240)\end{array}$ & $\begin{array}{l}0.0611^{* *} \\
(0.0294)\end{array}$ & $\begin{array}{r}-0.00186 \\
(0.0124)\end{array}$ \\
\hline EMP (base Employ & & & & & & & & & \\
\hline Others & $\begin{array}{c}-0.00973 \\
(0.0679)\end{array}$ & $\begin{array}{c}0.0616^{* * *} \\
(0.0226)\end{array}$ & $\begin{array}{l}-0.0108 \\
(0.0535)\end{array}$ & $\begin{array}{c}0.140^{* *} \\
(0.0621)\end{array}$ & $\begin{array}{l}-0.0469 \\
(0.0499)\end{array}$ & $\begin{array}{l}-0.0307 \\
(0.0490)\end{array}$ & $\begin{array}{c}0.0190 \\
(0.0579)\end{array}$ & $\begin{array}{l}-0.0449 \\
(0.0694)\end{array}$ & $\begin{array}{l}-0.0383 \\
(0.0280)\end{array}$ \\
\hline \multicolumn{10}{|l|}{ Sex (base male) } \\
\hline Female & $\begin{array}{l}0.122^{* *} \\
(0.0524)\end{array}$ & $\begin{array}{l}0.0529^{* *} \\
(0.0225)\end{array}$ & $\begin{array}{l}-0.155^{* *} \\
(0.0620)\end{array}$ & $\begin{array}{l}0.00698 \\
(0.0721)\end{array}$ & $\begin{array}{l}0.00877 \\
(0.0553)\end{array}$ & $\begin{array}{c}-0.00214 \\
(0.0516)\end{array}$ & $\begin{array}{c}0.0527 \\
(0.0477)\end{array}$ & $\begin{array}{l}-0.0134 \\
(0.0767)\end{array}$ & $\begin{array}{c}0.0630^{* * *} \\
(0.0186)\end{array}$ \\
\hline \multicolumn{10}{|c|}{ Marital (base single) } \\
\hline Others & $\begin{array}{c}0.0750 \\
(0.0661)\end{array}$ & $\begin{array}{l}0.0961^{* *} \\
(0.0382)\end{array}$ & $\begin{array}{c}-0.103^{* * *} \\
(0.0189)\end{array}$ & $\begin{array}{l}0.00925 \\
(0.0617)\end{array}$ & $\begin{array}{c}0.0158 \\
(0.0475)\end{array}$ & $\begin{array}{c}0.000633 \\
(0.0376)\end{array}$ & $\begin{array}{c}0.0559 \\
(0.0501)\end{array}$ & $\begin{array}{l}-0.0703 \\
(0.0618)\end{array}$ & $\begin{array}{l}0.0595^{* *} \\
(0.0296)\end{array}$ \\
\hline Children & $\begin{array}{l}-0.0176^{*} \\
(0.0105)\end{array}$ & $\begin{array}{l}0.0154^{* * *} \\
(0.00592)\end{array}$ & $\begin{array}{l}-0.00895 \\
(0.00753)\end{array}$ & $\begin{array}{c}-0.00457 \\
(0.0116)\end{array}$ & $\begin{array}{c}0.00408 \\
(0.00836)\end{array}$ & $\begin{array}{l}-6.56 \mathrm{e}-06 \\
(0.00805)\end{array}$ & $\begin{array}{c}0.0306^{* * *} \\
(0.0108)\end{array}$ & $\begin{array}{l}-0.0131 \\
(0.0111)\end{array}$ & $\begin{array}{c}0.00146 \\
(0.00427)\end{array}$ \\
\hline Observations & 1,616 & 1,616 & 1,616 & 1,616 & 1,616 & 1,616 & 1,616 & 1,616 & 1,616 \\
\hline
\end{tabular}

To capture the impact of the independent variables on the consumption level, the second-step equation (5) was estimated by OLS regression. Tables 5.1-5.2 show the results of the effects of the demographic characteristics and total expenditure for both households on the consumption level represented by the expenditure share of each commodity. The results show in general that most variables are statistically significant across many commodities for both groups. The inverse Mills ratio coefficients were all highly significant for all commodities, which implied that ignoring the censored bias problem would lead to inconsistent and biased estimates. Also, the household total expenditure coefficients were found to have a significant impact on expenditure shares for all commodities. However, the $\mathrm{HoH}$ sex coefficient was found to be insignificant for most commodities in the case of Kuwaiti households. In general, the results show the existence of the variation of the impact of household characteristics on consumption, indicating different magnitude effects on expenditure shares for both groups. 
Table 5.1. Second-Step Model Estimates for Kuwaiti Households

\begin{tabular}{|c|c|c|c|c|c|c|c|c|c|}
\hline VARIABLES & $\begin{array}{l}(1) \\
\text { Food }\end{array}$ & $\begin{array}{c}(2) \\
\text { Clothing }\end{array}$ & $\begin{array}{c}(3) \\
\text { Housing }\end{array}$ & $\begin{array}{c}(4) \\
\text { Health }\end{array}$ & $\begin{array}{l}(5) \\
\text { Trans. }\end{array}$ & $\begin{array}{c}(6) \\
\text { Comm. }\end{array}$ & $\begin{array}{l}(7) \\
\text { Rec. }\end{array}$ & $\begin{array}{l}(8) \\
\text { Res. }\end{array}$ & $\begin{array}{l}(9) \\
\text { Misc. }\end{array}$ \\
\hline EXP & $\begin{array}{c}0.0882^{* * *} \\
(0.0161)\end{array}$ & $\begin{array}{c}-0.0298^{* * *} \\
(0.00667)\end{array}$ & $\begin{array}{c}0.0428^{* * *} \\
(0.0121)\end{array}$ & $\begin{array}{l}0.0242^{* *} \\
(0.00962)\end{array}$ & $\begin{array}{l}0.173^{* * *} \\
(0.0248)\end{array}$ & $\begin{array}{c}0.0428^{* * *} \\
(0.0153)\end{array}$ & $\begin{array}{c}0.0264 \\
(0.0167)\end{array}$ & $\begin{array}{c}0.0412 \\
(0.0261)\end{array}$ & $\begin{array}{l}0.0320^{* * *} \\
(0.00904)\end{array}$ \\
\hline \multicolumn{10}{|l|}{ Size (base 1-2) } \\
\hline $3-5$ & $\begin{array}{l}-0.119^{* * *} \\
(0.0217)\end{array}$ & $\begin{array}{c}0.0321^{* * *} \\
(0.0123)\end{array}$ & $\begin{array}{c}-0.000660 \\
(0.0177)\end{array}$ & $\begin{array}{l}0.000614 \\
(0.00264)\end{array}$ & $\begin{array}{c}0.0167 \\
(0.0193)\end{array}$ & $\begin{array}{l}-0.0102^{*} \\
(0.00606)\end{array}$ & $\begin{array}{c}0.0130^{*} \\
(0.00689)\end{array}$ & $\begin{array}{l}-0.00781 \\
(0.00651)\end{array}$ & $\begin{array}{c}0.0168 \\
(0.0230)\end{array}$ \\
\hline $6-7$ & $\begin{array}{l}-0.0144 \\
(0.0209)\end{array}$ & $\begin{array}{l}0.0470^{* * * *} \\
(0.0126)\end{array}$ & $\begin{array}{c}-0.0859^{* * *} \\
(0.0182)\end{array}$ & $\begin{array}{l}-0.00278 \\
(0.00268)\end{array}$ & $\begin{array}{l}-0.0441^{* *} \\
(0.0196)\end{array}$ & $\begin{array}{l}-0.0196^{* *} \\
(0.00770)\end{array}$ & $\begin{array}{c}0.00851 \\
(0.00665)\end{array}$ & $\begin{array}{l}-0.0179 \\
(0.0133)\end{array}$ & $\begin{array}{c}0.0308 \\
(0.0236)\end{array}$ \\
\hline $8>$ & $\begin{array}{c}-0.0777^{* * *} \\
(0.0209)\end{array}$ & $\begin{array}{l}0.0580^{* * * *} \\
(0.0145)\end{array}$ & $\begin{array}{c}-0.0869^{* * *} \\
(0.0199)\end{array}$ & $\begin{array}{c}-0.00628^{* *} \\
(0.00290)\end{array}$ & $\begin{array}{l}0.0418^{* *} \\
(0.0208)\end{array}$ & $\begin{array}{l}-0.0129^{* *} \\
(0.00624)\end{array}$ & $\begin{array}{l}-0.000385 \\
(0.00983)\end{array}$ & $\begin{array}{l}0.000114 \\
(0.00758)\end{array}$ & $\begin{array}{l}-0.0180 \\
(0.0252)\end{array}$ \\
\hline \multicolumn{10}{|l|}{$\begin{array}{c}\text { Age (base } 20 \text { - } \\
\text { 39) }\end{array}$} \\
\hline $40-47$ & $\begin{array}{c}-0.0273^{* *} \\
(0.0139)\end{array}$ & $\begin{array}{l}-0.0269^{* *} \\
(0.0122)\end{array}$ & $\begin{array}{l}0.0213^{*} \\
(0.0120)\end{array}$ & $\begin{array}{l}0.00570^{* *} \\
(0.00227)\end{array}$ & $\begin{array}{l}0.0432^{* *} \\
(0.0171)\end{array}$ & $\begin{array}{l}-0.00342 \\
(0.00317)\end{array}$ & $\begin{array}{c}0.0111 \\
(0.00755)\end{array}$ & $\begin{array}{l}-0.00902 \\
(0.00676)\end{array}$ & $\begin{array}{l}-0.0257 \\
(0.0190)\end{array}$ \\
\hline $48-56$ & $\begin{array}{l}0.00367 \\
(0.0129)\end{array}$ & $\begin{array}{c}-0.0367^{* * *} \\
(0.0128)\end{array}$ & $\begin{array}{l}0.0453^{* * *} \\
(0.0140)\end{array}$ & $\begin{array}{c}-0.00853^{* * *} \\
(0.00281)\end{array}$ & $\begin{array}{c}-0.0482^{* * *} \\
(0.0182)\end{array}$ & $\begin{array}{l}-0.0179^{* * *} \\
(0.00600)\end{array}$ & $\begin{array}{c}0.00117 \\
(0.00678)\end{array}$ & $\begin{array}{l}-0.0334^{*} \\
(0.0179)\end{array}$ & $\begin{array}{l}0.00279 \\
(0.0198)\end{array}$ \\
\hline $57>$ & $\begin{array}{c}0.0156 \\
(0.0142)\end{array}$ & $\begin{array}{c}-0.0455^{* * *} \\
(0.0117)\end{array}$ & $\begin{array}{l}0.0427^{* * *} \\
(0.0144)\end{array}$ & $\begin{array}{c}-0.0135^{* * *} \\
(0.00396)\end{array}$ & $\begin{array}{l}-0.0182 \\
(0.0198)\end{array}$ & $\begin{array}{l}-0.0240^{* * *} \\
(0.00609)\end{array}$ & $\begin{array}{c}-0.0231^{* * *} \\
(0.00722)\end{array}$ & $\begin{array}{c}-0.0246^{* *} \\
(0.0119)\end{array}$ & $\begin{array}{c}0.0114 \\
(0.0209)\end{array}$ \\
\hline \multicolumn{10}{|l|}{$\begin{array}{c}\text { EDU (base } \\
\text { low) }\end{array}$} \\
\hline Moderate & $\begin{array}{l}-0.00743 \\
(0.00867)\end{array}$ & $\begin{array}{l}-0.0306^{* * *} \\
(0.00956)\end{array}$ & $\begin{array}{l}0.00402 \\
(0.0101)\end{array}$ & $\begin{array}{c}-0.00496^{* *} \\
(0.00214)\end{array}$ & $\begin{array}{l}-0.0324^{* *} \\
(0.0131)\end{array}$ & $\begin{array}{c}-0.00727^{* *} \\
(0.00285)\end{array}$ & $\begin{array}{l}0.0162^{* *} \\
(0.00817)\end{array}$ & $\begin{array}{c}-0.0127 \\
(0.00811)\end{array}$ & $\begin{array}{l}0.00673 \\
(0.0141)\end{array}$ \\
\hline High & $\begin{array}{l}-0.0229^{*} \\
(0.0120)\end{array}$ & $\begin{array}{c}-0.0575^{* * *} \\
(0.0110)\end{array}$ & $\begin{array}{l}0.0304^{* *} \\
(0.0128)\end{array}$ & $\begin{array}{c}-0.0138^{* * *} \\
(0.00388)\end{array}$ & $\begin{array}{l}-0.0248^{*} \\
(0.0138)\end{array}$ & $\begin{array}{l}-0.0276^{* * *} \\
(0.00983)\end{array}$ & $\begin{array}{l}-0.0175^{* *} \\
(0.00842)\end{array}$ & $\begin{array}{l}-0.0163 \\
(0.0132)\end{array}$ & $\begin{array}{c}0.0160 \\
(0.0167)\end{array}$ \\
\hline \multicolumn{10}{|l|}{$\begin{array}{l}\text { EMP (base } \\
\text { Employed) }\end{array}$} \\
\hline Others & $\begin{array}{c}-0.0572^{* * *} \\
(0.0116)\end{array}$ & $\begin{array}{l}-0.00274 \\
(0.00910)\end{array}$ & $\begin{array}{l}0.0354^{* * *} \\
(0.0113)\end{array}$ & $\begin{array}{l}0.0184^{* * *} \\
(0.00548)\end{array}$ & $\begin{array}{l}0.0328^{* *} \\
(0.0150)\end{array}$ & $\begin{array}{l}0.0177^{* * *} \\
(0.00387)\end{array}$ & $\begin{array}{c}0.0133 \\
(0.00870)\end{array}$ & $\begin{array}{c}0.00341 \\
(0.00454)\end{array}$ & $\begin{array}{l}-0.0237 \\
(0.0162)\end{array}$ \\
\hline \multicolumn{10}{|l|}{$\begin{array}{l}\text { Sex (base } \\
\text { male) }\end{array}$} \\
\hline Female & $\begin{array}{l}0.00120 \\
(0.0246)\end{array}$ & $\begin{array}{l}0.0309^{*} \\
(0.0185)\end{array}$ & $\begin{array}{l}-0.0133 \\
(0.0190)\end{array}$ & $\begin{array}{c}0.00347 \\
(0.00301)\end{array}$ & $\begin{array}{l}-0.115^{* * *} \\
(0.0227)\end{array}$ & $\begin{array}{c}0.00415 \\
(0.00537)\end{array}$ & $\begin{array}{c}0.0111 \\
(0.00728)\end{array}$ & $\begin{array}{l}-0.00115 \\
(0.00473)\end{array}$ & $\begin{array}{l}0.00205 \\
(0.0273)\end{array}$ \\
\hline \multicolumn{10}{|l|}{$\begin{array}{l}\text { Marital (base } \\
\text { single) }\end{array}$} \\
\hline Others & $\begin{array}{c}0.0148 \\
(0.0248)\end{array}$ & $\begin{array}{c}0.0533^{* * *} \\
(0.0175)\end{array}$ & $\begin{array}{l}-0.00221 \\
(0.0180)\end{array}$ & $\begin{array}{c}0.00160 \\
(0.00253)\end{array}$ & $\begin{array}{l}-0.0302 \\
(0.0218)\end{array}$ & $\begin{array}{c}0.0139^{*} \\
(0.00730)\end{array}$ & $\begin{array}{l}0.0333^{* *} \\
(0.0151)\end{array}$ & $\begin{array}{c}0.0140 \\
(0.0109)\end{array}$ & $\begin{array}{l}-0.0236 \\
(0.0274)\end{array}$ \\
\hline Children & $\begin{array}{c}-0.00414^{* *} \\
(0.00168)\end{array}$ & $\begin{array}{c}-0.00503^{* * *} \\
(0.00180)\end{array}$ & $\begin{array}{l}0.0109^{* * *} \\
(0.00248)\end{array}$ & $\begin{array}{l}0.00171^{* * *} \\
(0.000645)\end{array}$ & $\begin{array}{c}-0.00847^{* * *} \\
(0.00276)\end{array}$ & $\begin{array}{l}0.00279^{* * *} \\
(0.000913)\end{array}$ & $\begin{array}{l}0.00582^{* *} \\
(0.00245)\end{array}$ & $\begin{array}{c}0.00245 \\
(0.00158)\end{array}$ & $\begin{array}{c}0.00410 \\
(0.00322)\end{array}$ \\
\hline Mills & $\begin{array}{c}0.781^{* * *} \\
(0.100)\end{array}$ & $\begin{array}{l}0.308^{* * *} \\
(0.0342)\end{array}$ & $\begin{array}{l}0.852^{* * *} \\
(0.0599)\end{array}$ & $\begin{array}{l}0.107^{* * *} \\
(0.0322)\end{array}$ & $\begin{array}{l}0.789^{* * *} \\
(0.110)\end{array}$ & $\begin{array}{l}0.259^{* * *} \\
(0.0809)\end{array}$ & $\begin{array}{c}0.204^{* *} \\
(0.0889)\end{array}$ & $\begin{array}{l}0.207^{*} \\
(0.112)\end{array}$ & $\begin{array}{l}0.338^{* * *} \\
(0.0641)\end{array}$ \\
\hline Constant & $\begin{array}{c}-0.848^{* * *} \\
(0.169)\end{array}$ & $\begin{array}{l}0.310^{* * *} \\
(0.0628)\end{array}$ & $\begin{array}{c}-0.349^{* * *} \\
(0.115)\end{array}$ & $\begin{array}{c}-0.269^{* *} \\
(0.107)\end{array}$ & $\begin{array}{c}-1.520^{* * *} \\
(0.231)\end{array}$ & $\begin{array}{c}-0.421^{* * *} \\
(0.152)\end{array}$ & $\begin{array}{l}-0.313 \\
(0.191)\end{array}$ & $\begin{array}{l}-0.414 \\
(0.270)\end{array}$ & $\begin{array}{c}0.195^{* *} \\
(0.0818)\end{array}$ \\
\hline Observations & 795 & 983 & 910 & 541 & 857 & 806 & 784 & 690 & 1,016 \\
\hline R-squared & 0.376 & 0.408 & 0.577 & 0.414 & 0.235 & 0.461 & 0.270 & 0.266 & 0.065 \\
\hline
\end{tabular}

Robust standard errors in parentheses. ${ }^{* *} \mathrm{p}<0.01,{ }^{* *} \mathrm{p}<0.05,{ }^{*} \mathrm{p}<0.1$ 
Table 5.2. Second-Step Model Estimates for Expatriate Households

\begin{tabular}{|c|c|c|c|c|c|c|c|c|c|}
\hline VARIABLES & $\begin{array}{c}(1) \\
\text { Food }\end{array}$ & $\begin{array}{c}(2) \\
\text { Clothing }\end{array}$ & $\begin{array}{c}\text { (3) } \\
\text { Housing }\end{array}$ & $\begin{array}{c}(4) \\
\text { Health }\end{array}$ & $\begin{array}{c}\text { (5) } \\
\text { Trans. }\end{array}$ & $\begin{array}{c}(6) \\
\text { Comm. }\end{array}$ & $\begin{array}{l}(7) \\
\text { Rec. }\end{array}$ & $\begin{array}{l}(8) \\
\text { Res. }\end{array}$ & $\begin{array}{c}\text { (9) } \\
\text { Misc. }\end{array}$ \\
\hline EXP & $\begin{array}{c}0.0850^{* * *} \\
(0.0182)\end{array}$ & $\begin{array}{c}0.00399 \\
(0.00543)\end{array}$ & $\begin{array}{l}-0.0122 \\
(0.0113)\end{array}$ & $\begin{array}{c}0.0506 \\
(0.0341)\end{array}$ & $\begin{array}{l}0.123^{* * *} \\
(0.0161)\end{array}$ & $\begin{array}{c}0.0533^{* * *} \\
(0.0124)\end{array}$ & $\begin{array}{l}0.0232^{* * *} \\
(0.00688)\end{array}$ & $\begin{array}{l}0.141^{* * *} \\
(0.0336)\end{array}$ & $\begin{array}{l}0.0344^{* * *} \\
(0.00828)\end{array}$ \\
\hline \multicolumn{10}{|l|}{ Size (base 1-2) } \\
\hline $3-5$ & $\begin{array}{c}0.0867^{* * *} \\
(0.0119)\end{array}$ & $\begin{array}{l}0.0130^{* *} \\
(0.00575)\end{array}$ & $\begin{array}{c}-0.0755^{* * *} \\
(0.0106)\end{array}$ & $\begin{array}{l}-0.00422 \\
(0.00362)\end{array}$ & $\begin{array}{c}0.00780 \\
(0.00773)\end{array}$ & $\begin{array}{l}-0.00410 \\
(0.00462)\end{array}$ & $\begin{array}{l}0.0123^{* * *} \\
(0.00382)\end{array}$ & $\begin{array}{l}0.0179^{* * *} \\
(0.00459)\end{array}$ & $\begin{array}{c}-0.00596 \\
(0.0116)\end{array}$ \\
\hline $6-7$ & $\begin{array}{l}0.121^{* * *} \\
(0.0170)\end{array}$ & $\begin{array}{l}-0.00204 \\
(0.00962)\end{array}$ & $\begin{array}{c}-0.0727^{* * *} \\
(0.0174)\end{array}$ & $\begin{array}{l}-0.0179 \\
(0.0110)\end{array}$ & $\begin{array}{l}-0.0284^{*} \\
(0.0148)\end{array}$ & $\begin{array}{l}-0.0249^{* * *} \\
(0.00824)\end{array}$ & $\begin{array}{c}0.0134^{*} \\
(0.00721)\end{array}$ & $\begin{array}{c}0.0125^{* *} \\
(0.00520)\end{array}$ & $\begin{array}{c}-0.00513 \\
(0.0189)\end{array}$ \\
\hline $8>$ & $\begin{array}{l}0.118^{* * *} \\
(0.0218)\end{array}$ & $\begin{array}{c}0.0110 \\
(0.0157)\end{array}$ & $\begin{array}{c}0.0298 \\
(0.0243)\end{array}$ & $\begin{array}{l}-0.00706 \\
(0.00513)\end{array}$ & $\begin{array}{c}0.0207 \\
(0.0256)\end{array}$ & $\begin{array}{c}-0.0451^{* * *} \\
(0.0156)\end{array}$ & $\begin{array}{c}-0.0243^{* *} \\
(0.0111)\end{array}$ & $\begin{array}{c}0.0108 \\
(0.00907)\end{array}$ & $\begin{array}{c}-0.0690^{* *} \\
(0.0306)\end{array}$ \\
\hline \multicolumn{10}{|l|}{ Age (base 20 -39) } \\
\hline $40-47$ & $\begin{array}{c}-0.0249^{* * *} \\
(0.00891)\end{array}$ & $\begin{array}{l}-0.0186^{* * *} \\
(0.00583)\end{array}$ & $\begin{array}{c}0.0280^{* * *} \\
(0.0101)\end{array}$ & $\begin{array}{l}-0.0194^{*} \\
(0.0100)\end{array}$ & $\begin{array}{l}-0.0141^{*} \\
(0.00812)\end{array}$ & $\begin{array}{l}0.00586^{*} \\
(0.00342)\end{array}$ & $\begin{array}{l}0.00709^{*} \\
(0.00388)\end{array}$ & $\begin{array}{l}-0.0308^{* * *} \\
(0.00733)\end{array}$ & $\begin{array}{c}-0.00953 \\
(0.0110)\end{array}$ \\
\hline $48-56$ & $\begin{array}{c}0.00823 \\
(0.00943)\end{array}$ & $\begin{array}{l}-0.00987 \\
(0.00628)\end{array}$ & $\begin{array}{c}-0.0463^{* * *} \\
(0.0108)\end{array}$ & $\begin{array}{c}-0.00847^{* *} \\
(0.00342)\end{array}$ & $\begin{array}{c}-0.0232^{* * *} \\
(0.00789)\end{array}$ & $\begin{array}{l}-0.0145^{* * *} \\
(0.00386)\end{array}$ & $\begin{array}{c}0.00304 \\
(0.00463)\end{array}$ & $\begin{array}{l}-0.101^{* * *} \\
(0.0235)\end{array}$ & $\begin{array}{l}0.00617 \\
(0.0122)\end{array}$ \\
\hline $57>$ & $\begin{array}{c}0.0425^{* * *} \\
(0.0106)\end{array}$ & $\begin{array}{c}0.00745 \\
(0.00690)\end{array}$ & $\begin{array}{c}-0.0302^{* *} \\
(0.0126)\end{array}$ & $\begin{array}{c}-0.00669^{* *} \\
(0.00325)\end{array}$ & $\begin{array}{l}-0.0147^{*} \\
(0.00855)\end{array}$ & $\begin{array}{l}0.00981^{*} \\
(0.00566)\end{array}$ & $\begin{array}{l}-0.00264 \\
(0.00450)\end{array}$ & $\begin{array}{c}-0.0854^{* * *} \\
(0.0192)\end{array}$ & $\begin{array}{l}-0.00342 \\
(0.0139)\end{array}$ \\
\hline \multicolumn{10}{|l|}{ EDU (base low) } \\
\hline Moderate & $\begin{array}{l}-0.00452 \\
(0.00873)\end{array}$ & $\begin{array}{l}-0.00853 \\
(0.00599)\end{array}$ & $\begin{array}{c}-0.0318^{* * *} \\
(0.0106)\end{array}$ & $\begin{array}{l}-0.0119^{*} \\
(0.00709)\end{array}$ & $\begin{array}{c}0.0156^{*} \\
(0.00865)\end{array}$ & $\begin{array}{l}-0.0184^{* * *} \\
(0.00531)\end{array}$ & $\begin{array}{l}0.0191^{* * *} \\
(0.00367)\end{array}$ & $\begin{array}{c}0.0729^{* * *} \\
(0.0185)\end{array}$ & $\begin{array}{l}0.00956 \\
(0.0119)\end{array}$ \\
\hline High & $\begin{array}{c}0.0148^{*} \\
(0.00831)\end{array}$ & $\begin{array}{l}-0.00649 \\
(0.00575)\end{array}$ & $\begin{array}{c}-0.0460^{* * *} \\
(0.0103)\end{array}$ & $\begin{array}{c}-0.00513^{* *} \\
(0.00260)\end{array}$ & $\begin{array}{l}-0.000347 \\
(0.00750)\end{array}$ & $\begin{array}{l}-0.0287^{* * *} \\
(0.00592)\end{array}$ & $\begin{array}{l}0.0335^{* * *} \\
(0.00482)\end{array}$ & $\begin{array}{c}0.0509^{* * *} \\
(0.0133)\end{array}$ & $\begin{array}{c}0.0150 \\
(0.0111)\end{array}$ \\
\hline \multicolumn{10}{|l|}{$\begin{array}{l}\text { EMP (base } \\
\text { Employed) }\end{array}$} \\
\hline Others & $\begin{array}{c}-0.0615^{* * *} \\
(0.0212)\end{array}$ & $\begin{array}{c}0.0137 \\
(0.0182)\end{array}$ & $\begin{array}{c}0.0122 \\
(0.0270)\end{array}$ & $\begin{array}{l}0.0297^{* *} \\
(0.0151)\end{array}$ & $\begin{array}{l}-0.00354 \\
(0.0271)\end{array}$ & $\begin{array}{l}-0.0158^{*} \\
(0.00848)\end{array}$ & $\begin{array}{c}0.00875 \\
(0.00800)\end{array}$ & $\begin{array}{c}-0.0361^{* * *} \\
(0.0119)\end{array}$ & $\begin{array}{l}-0.0179 \\
(0.0344)\end{array}$ \\
\hline \multicolumn{10}{|l|}{ Sex (base male) } \\
\hline Female & $\begin{array}{l}0.182^{* * *} \\
(0.0342)\end{array}$ & $\begin{array}{l}0.0282^{*} \\
(0.0149)\end{array}$ & $\begin{array}{l}-0.175^{* * *} \\
(0.0300)\end{array}$ & $\begin{array}{l}0.000433 \\
(0.00417)\end{array}$ & $\begin{array}{l}-0.00855 \\
(0.0163)\end{array}$ & $\begin{array}{c}0.00707 \\
(0.00659)\end{array}$ & $\begin{array}{l}0.0185^{* *} \\
(0.00724)\end{array}$ & $\begin{array}{l}-0.0209^{* *} \\
(0.00907)\end{array}$ & $\begin{array}{l}0.0877^{* *} \\
(0.0354)\end{array}$ \\
\hline \multicolumn{10}{|l|}{$\begin{array}{l}\text { Marital (base } \\
\text { single) }\end{array}$} \\
\hline Others & $\begin{array}{c}0.0838^{* * *} \\
(0.0283)\end{array}$ & $\begin{array}{l}0.0267^{*} \\
(0.0145)\end{array}$ & $\begin{array}{l}-0.190^{* * *} \\
(0.0295)\end{array}$ & $\begin{array}{l}0.00707^{* *} \\
(0.00346)\end{array}$ & $\begin{array}{l}-0.00809 \\
(0.0162)\end{array}$ & $\begin{array}{l}0.00859^{*} \\
(0.00459)\end{array}$ & $\begin{array}{l}0.0218^{* * *} \\
(0.00723)\end{array}$ & $\begin{array}{c}-0.0646^{* * *} \\
(0.0156)\end{array}$ & $\begin{array}{c}0.0820^{* * *} \\
(0.0303)\end{array}$ \\
\hline Mills & $\begin{array}{c}0.936^{* * *} \\
(0.129)\end{array}$ & $\begin{array}{l}0.312^{* * *} \\
(0.0390)\end{array}$ & $\begin{array}{l}0.640^{* * *} \\
(0.0525)\end{array}$ & $\begin{array}{c}0.150^{*} \\
(0.0874)\end{array}$ & $\begin{array}{l}0.484^{* * *} \\
(0.0647)\end{array}$ & $\begin{array}{l}0.300^{* * *} \\
(0.0596)\end{array}$ & $\begin{array}{l}0.166^{* * *} \\
(0.0268)\end{array}$ & $\begin{array}{c}0.550^{* * *} \\
(0.124)\end{array}$ & $\begin{array}{l}0.161^{* * *} \\
(0.0442)\end{array}$ \\
\hline Children & $\begin{array}{l}-0.0241^{* * *} \\
(0.00388)\end{array}$ & $\begin{array}{c}0.00647^{* * *} \\
(0.00250)\end{array}$ & $\begin{array}{l}-0.00682 \\
(0.00421)\end{array}$ & $\begin{array}{l}-0.000682 \\
(0.000829)\end{array}$ & $\begin{array}{l}-0.00344 \\
(0.00335)\end{array}$ & $\begin{array}{l}-0.00281^{*} \\
(0.00157)\end{array}$ & $\begin{array}{l}0.0109^{* * *} \\
(0.00182)\end{array}$ & $\begin{array}{l}-0.0122^{* * *} \\
(0.00301)\end{array}$ & $\begin{array}{l}0.000815 \\
(0.00481)\end{array}$ \\
\hline Constant & $\begin{array}{c}-1.037^{* * *} \\
(0.208)\end{array}$ & $\begin{array}{c}-0.000641 \\
(0.0517)\end{array}$ & $\begin{array}{l}0.489^{* * *} \\
(0.0958)\end{array}$ & $\begin{array}{l}-0.487 \\
(0.333)\end{array}$ & $\begin{array}{c}-1.054^{* * *} \\
(0.148)\end{array}$ & $\begin{array}{c}-0.451^{* * *} \\
(0.109)\end{array}$ & $\begin{array}{c}-0.266^{* * *} \\
(0.0694)\end{array}$ & $\begin{array}{c}-1.386^{* * *} \\
(0.336)\end{array}$ & $\begin{array}{c}0.110 \\
(0.0747)\end{array}$ \\
\hline Observations & 1,191 & 1,420 & 1,345 & 743 & 1,206 & 1,241 & 1,134 & 880 & 1,445 \\
\hline R-squared & 0.452 & 0.429 & 0.570 & 0.321 & 0.258 & 0.490 & 0.314 & 0.471 & 0.036 \\
\hline
\end{tabular}

Robust standard errors in parentheses. ${ }^{* *} \mathrm{p}<0.01,{ }^{* *} \mathrm{p}<0.05,{ }^{*} \mathrm{p}<0.1$.

The empirical results indicate that food expenditure shares for the Kuwaiti households decrease with size, while expatriate households exhibit the opposite effect. This finding raises the question of how Kuwaiti households achieve economies of scale in the consumption of food products, the most obvious explanation of which is the effect of food subsidy programmes in Kuwait, which are accorded only to Kuwaiti households, and which vary according to family size (i.e. the larger the household, the greater the value of subsidies obtained). Also, the results show a reverse effect of the educational level between the two groups on the consumption of food. The more highly educated Kuwaiti household 
consumes less than their moderately and low educated counterparts do, whereas the educational level for expatriates is positively related to the consumption level.

Also, the Kuwaiti households display a significant negative impact on the level of consuming clothing, while expatriates show a positive impact. Another significant difference is the total expenditure on housing, which was observed to be negative for expatriates and positive for Kuwaitis, whereby (holding everything else constant) a unit increase in total household expenditure affects the level of consumption by increasing the expenditure share on housing for Kuwaitis by $4.3 \%$, compared to a decrease of $1.2 \%$ for expatriates. The effects of age were also statistically significant between the two groups. It was observed that older Kuwaiti households spend more on housing and older expatriates spend less. Apart from that, the higher the educational level for expatriates, the less they spend on housing, while highly educated Kuwaitis spend more. The opposite results were found for the consumption of transportation and recreation goods and services, with moderately educated expatriates spending more than their less-educated peers, and moderately educated Kuwaitis spending less.

Similarly, the consumption of communication commodities showed a variation in household characteristics between the two groups. It was found that Kuwaitis aged over 65 spend less than younger households but expatriates spend more. Also, it was found that the employment status of the household and the number of children impacts the expenditure shares positively for Kuwaitis and negatively for expatriates. Overall, Tables 5.1-5.2 show the dissimilarity of the marginal effects between the two types of household's characteristics and total expenditure on their consumption level. These effects reflect the differences in ethnic, cultural backgrounds and preferences of the two groups towards expenditure.

To test the robustness of the estimated coefficients between the two households, the Chow test was conducted. The null hypothesis for the test suggests that the estimated coefficients of the independent variables from the Kuwaiti and expatriate households are equal. The results of the Chow test are reported in Table 6. The decision to reject the null hypothesis is reported as a yes whereas no indicates the failure to reject the null hypothesis. For most commodities, the results show the estimated coefficients from the model are statistically significant and differences between the two household types exist. Therefore, the results confirm the existence of different consumption patterns between the two households groups and the treatment of the two groups as a combined sample will yield distorted results.

Table 6. Chow Test results

\begin{tabular}{ccc}
\hline Commodity Groups & F-value & Decision \\
\hline Food and non-alcoholic beverages & $4.404^{* * *}$ & Yes \\
Clothing and footwear & $21.61^{* * *}$ & Yes \\
fuels) & -1.966 & No \\
Health & $3.122^{* * *}$ & Yes \\
Transport & $52.189^{* * *}$ & Yes \\
Communication & $11.527^{* * *}$ & Yes \\
Recreation and culture & -1.288 & No \\
Restaurants and hotels & $2.67^{* * *}$ & Yes \\
Miscellaneous goods and services & 0.399 & No \\
\hline
\end{tabular}

Note: ${ }^{* *}$ indicates significance at 1 percent level

Also, to test the variation in consumption between the expatriates and Kuwaitis, a pooled regression was estimated. Following the Heckman two-step estimation procedure, the estimation of equation (4) was estimated, with the addition of a dummy variable (nat), which takes the value of one for expatriates and zero for Kuwaitis. Therefore, the difference in expenditure between the two groups 
will be reflected by the sign and the magnitude of the dummy coefficient. If there are no differences, the coefficients of the dummy variable should equal zero. The results from the pooled regression are reported in Appendix 1, which shows that the coefficients of the dummy variables were significant at the 1 percent level for all commodities; except for transportation and miscellaneous goods and services, the results were insignificant.

As a robustness check, the model was modified by changing some of the regressors specifications from categorical variables to continues. To pinpoint this, we re-estimate our model including the logarithm of two variables Size and Age. Results are shown in appendix 2 and 3. For both types of households, the results are the same in terms of signs and statistical significance. Nevertheless, in terms of point estimates, there are minor changes in the magnitudes across all commodity groups except for miscellaneous goods. In general, the robustness checks confirm our earlier findings that our model is robust in terms of signs and level of significance.

Overall, the first step model results showed the different effects of household characteristics on the probability of consumption, while the second step model has shown the impact of those variables on the level of consumption. Both results displayed different signs and magnitudes effects between the two types of households. These findings indicate significant differences in consumption patterns between the two groups, influenced by the variation in social status, lifestyles, and preferences of the households.

In Table 7 the expenditure elasticities are reported. Following equation (2), expenditure elasticities were computed using the estimates from the second-step model. Most of the expenditure elasticities were statistically significant at the $1 \%$ level for both households. The results show that almost all commodities are considered as luxury goods, with an elasticity higher than one. Clothing for the case of Kuwaitis and housing for expatriates were considered as necessities, with an elasticity of less than one. The expenditure elasticities for most commodities are somewhat consistent with the literature. Except for food, most studies conclude that food is a necessity good. However, it was expected for the case of Kuwait to appear as a luxury good, since the most prolifically consumed food products are meat, fish, rice, and chicken, which were found to be luxury goods in prior international studies (Zhang and Goddard, 2010; Jabarin, 2005; Ben Kaabia and Gil, 2001).

The expenditure elasticities between the two household types differ across all commodities with the single exception of miscellaneous goods and services. Kuwaiti households classify housing as a luxury good, while expatriates consider it a necessity; conversely, expatriates view clothing as a luxury commodity while Kuwaitis consider them as a necessity. These results reflect the importance of social prestige in Kuwait, where ostentatious displays of wealth and power are de rigueur for nationals, entailing lavish spending on housing, parties, and clothing, whereas most expatriates aim to live a frugal lifestyle and amass a capital sum to return to their home countries (Alomar, 2015).

The most highly sensitive commodities were communication for Kuwaitis, with an elasticity of 3.2 , and restaurants and hotels for expatriates, with an elasticity of 6.7, the highest value in the estimated elasticities. The least sensitive commodities are those classified as necessity goods. The results confirm that the effects of changes in total expenditure (income) of the households are entirely different between the two groups. Also, these results confirm the estimates obtained in Tables 4.1-4.2 in that there are differences in the magnitudes of the marginal effects of total expenditure on the expenditure shares of commodities. 
Table 7. Expenditure Elasticities for Kuwaiti and Expatriate Households

\begin{tabular}{ccc}
\hline Commodity Groups & Kuwaiti & Expatriate \\
\hline Food and non-alcoholic beverages & $1.8^{* * *}$ & $1.7^{* * *}$ \\
Clothing and footwear & $0.7^{* * *}$ & $1.1^{2}$ \\
Housing (includes water, electricity, gas and & $1.2^{* * *}$ & $0.8^{3}$ \\
other fuels) & $2.7^{* *}$ & $3.6^{4}$ \\
Health & $2.7^{* * *}$ & $3.0^{* * *}$ \\
Transport & $3.2^{* * *}$ & $2.6^{* * *}$ \\
Communication & 1.7 & $1.5^{* * *}$ \\
Recreation and culture & 2.7 & $6.7^{* * *}$ \\
Restaurants and hotels & $1.1^{* * *}$ & $1.1^{* * *}$ \\
\hline Miscellaneous goods and services & &
\end{tabular}

Source: Authors Calculation

Note: ${ }^{* *}$ and ${ }^{* *}$ indicate significance at 1 and 5 percent level, respectively

\section{Conclusions}

This paper utilised Kuwait Household Expenditure Survey (2013) data to analyse household expenditure patterns in Kuwait. Given the unique structure of the Kuwaiti population, the analysis was conducted on Kuwaiti and expatriate households separately, for comparative purposes. The primary objectives of this paper were to investigate and compare the differences in consumption behaviour for Kuwaiti and expatriate households by examining the impact of household characteristics on the expenditure level on nine commodities. To achieve the study objectives and provide a better understanding of expenditure patterns, the expenditure elasticities and the impact of household characteristics on expenditure level was estimated. The Working-Leser Engle curve model was applied as an estimation procedure as it is consistent with the consumer demand theory. The Heckman two-step method was applied to account for the censored biased estimates. In general, the results revealed that expenditure patterns between Kuwaitis and expatriates differ significantly. The results of the marginal effects of household characteristics and total expenditure on expenditure patterns are substantially different between Kuwaitis and expatriates. The estimated expenditure elasticities for nine commodities were found to be statistically significant at the one percent level. The elasticity scales varied between the two sample groups. These findings pose several policy implications. Policymakers can see the likely impact of implementing changes in prices or wages on the demand goods for two different population groups. Also, due to the high sensitivity of demand towards food and housing for Kuwaitis, the government should consider restructuring the subsidies programs in these two commodity groups. Additionally, the results can be used to assess the effects of imposing new immigration policies on expatriate households by relating economic impacts on household characteristics, and the effect of policies on consumption levels. Examples of such policies that have been imposed before include restricting the number of dependent siblings and deportation.

This paper is considered the first attempt to incorporate different demographic factors in the analysis of household expenditure patterns in Kuwait and deriving expenditure elasticities for several commodities. However, it suffers from several limitations that can be tackled in future research. The

\footnotetext{
2 Expenditure elasticity for clothing is a puzzling matter that needs further study.

${ }^{3}$ By law in Kuwait expatriates are forbidden from owning houses this has its effect on the significance of the elasticity of housing as it only reflects the utilities.

${ }^{4}$ Elasticity for health is insignificant due to the large number of low skilled labour employed in Kuwait, which cannot afford the expenses of health services. Another reason can be the high number of zero/missing observations.
} 
problem of endogeneity within the model and cross-price elasticities of commodities should be considered in future research.

Acknowledgements: We would like to thank Dr. Ahmed Alawadhi for his insightful comments that benefited the paper.

Author Contributions: Conceptualization, M.A.; methodology, M.A. and A.A.; software, M.A. and A.N.; validation, M.A. and A.A.; formal analysis, M.A.; investigation, A.A.; resources, M.A.; data curation, A.N.; writing-original draft preparation, M.A.; writing-review and editing, A.A. and A.N.; visualization, A.A.; supervision, M.A.; project administration, M.A.

Funding: This research received no external funding.

Conflicts of Interest: The authors declare no conflict of interest.

Appendix 1. Pooled Regression

\begin{tabular}{|c|c|c|c|c|c|c|c|c|c|}
\hline VARIABLES & $\begin{array}{c}(1) \\
\text { Food }\end{array}$ & $\begin{array}{c}(2) \\
\text { Clothing }\end{array}$ & $\begin{array}{c}\text { (3) } \\
\text { Housing }\end{array}$ & $\begin{array}{c}(4) \\
\text { Health }\end{array}$ & $\begin{array}{l}\text { (5) } \\
\text { Trans. }\end{array}$ & $\begin{array}{c}(6) \\
\text { Comm. }\end{array}$ & $\begin{array}{l}(7) \\
\text { Rec. }\end{array}$ & $\begin{array}{l}(8) \\
\text { Res. }\end{array}$ & $\begin{array}{c}(9) \\
\text { Misc. }\end{array}$ \\
\hline EXP & $\begin{array}{c}0.0974^{* * *} \\
(0.0122)\end{array}$ & $\begin{array}{c}-0.00897^{* *} \\
(0.00429)\end{array}$ & $\begin{array}{l}0.0281^{* * *} \\
(0.00818)\end{array}$ & $\begin{array}{l}0.0399^{*} \\
(0.0207)\end{array}$ & $\begin{array}{l}0.146^{* * *} \\
(0.0142)\end{array}$ & $\begin{array}{c}0.0494^{* * *} \\
(0.0101)\end{array}$ & $\begin{array}{l}0.0285^{* * *} \\
(0.00824)\end{array}$ & $\begin{array}{c}0.0791^{* * *} \\
(0.0198)\end{array}$ & $\begin{array}{l}0.0343^{* * *} \\
(0.00604)\end{array}$ \\
\hline \multicolumn{10}{|l|}{ Size (base 1-2) } \\
\hline $3-5$ & $\begin{array}{l}0.0225^{* * *} \\
(0.00742)\end{array}$ & $\begin{array}{l}0.0277^{* * *} \\
(0.00498)\end{array}$ & $\begin{array}{c}-0.0792^{* * *} \\
(0.00833)\end{array}$ & $\begin{array}{c}-0.00463^{* *} \\
(0.00222)\end{array}$ & $\begin{array}{c}0.0104 \\
(0.00667)\end{array}$ & $\begin{array}{c}-0.0118^{* * *} \\
(0.00386)\end{array}$ & $\begin{array}{l}0.0200^{* * *} \\
(0.00374)\end{array}$ & $\begin{array}{l}-0.00221 \\
(0.00293)\end{array}$ & $\begin{array}{l}-0.00320 \\
(0.00940)\end{array}$ \\
\hline $6-7$ & $\begin{array}{c}0.0749^{* * *} \\
(0.0111)\end{array}$ & $\begin{array}{l}0.0291^{* * *} \\
(0.00713)\end{array}$ & $\begin{array}{c}-0.0998^{* * *} \\
(0.0115)\end{array}$ & $\begin{array}{l}-0.0149^{* *} \\
(0.00620)\end{array}$ & $\begin{array}{c}-0.0403^{* * *} \\
(0.0107)\end{array}$ & $\begin{array}{c}-0.0338^{* * *} \\
(0.00672)\end{array}$ & $\begin{array}{l}0.0158^{* * *} \\
(0.00491)\end{array}$ & $\begin{array}{c}-0.0212^{* * *} \\
(0.00681)\end{array}$ & $\begin{array}{c}-0.00144 \\
(0.0132)\end{array}$ \\
\hline $8>$ & $\begin{array}{l}0.0279^{* *} \\
(0.0115)\end{array}$ & $\begin{array}{l}0.0359^{* * *} \\
(0.00940)\end{array}$ & $\begin{array}{c}-0.0586^{* * *} \\
(0.0136)\end{array}$ & $\begin{array}{l}-0.0132^{* *} \\
(0.00553)\end{array}$ & $\begin{array}{l}0.0294^{* *} \\
(0.0145)\end{array}$ & $\begin{array}{c}-0.0306^{* * *} \\
(0.00758)\end{array}$ & $\begin{array}{l}-0.00730 \\
(0.00669)\end{array}$ & $\begin{array}{c}0.00105 \\
(0.00496)\end{array}$ & $\begin{array}{c}-0.0509^{* * *} \\
(0.0167)\end{array}$ \\
\hline \multicolumn{10}{|l|}{ Age (base 20 -39) } \\
\hline $40-47$ & $\begin{array}{l}-0.0272^{* * *} \\
(0.00735)\end{array}$ & $\begin{array}{c}-0.0223^{* * *} \\
(0.00555)\end{array}$ & $\begin{array}{l}0.0230^{* * *} \\
(0.00775)\end{array}$ & $\begin{array}{l}-0.00961^{* *} \\
(0.00392)\end{array}$ & $\begin{array}{c}0.00261 \\
(0.00745)\end{array}$ & $\begin{array}{c}0.00411 \\
(0.00258)\end{array}$ & $\begin{array}{c}0.00896^{* * *} \\
(0.00325)\end{array}$ & $\begin{array}{c}-0.0169^{* * *} \\
(0.00467)\end{array}$ & $\begin{array}{c}-0.0143 \\
(0.00958)\end{array}$ \\
\hline $48-56$ & $\begin{array}{c}0.00798 \\
(0.00757)\end{array}$ & $\begin{array}{c}-0.0168^{* * *} \\
(0.00595)\end{array}$ & $\begin{array}{l}-0.0231^{* * *} \\
(0.00836)\end{array}$ & $\begin{array}{c}-0.00884^{* * *} \\
(0.00280)\end{array}$ & $\begin{array}{c}-0.0297^{* * *} \\
(0.00757)\end{array}$ & $\begin{array}{c}-0.0137^{* * *} \\
(0.00330)\end{array}$ & $\begin{array}{c}0.00298 \\
(0.00372)\end{array}$ & $\begin{array}{c}-0.0604^{* * *} \\
(0.0143)\end{array}$ & $\begin{array}{l}0.00447 \\
(0.0102)\end{array}$ \\
\hline $57>$ & $\begin{array}{l}0.0312^{* * *} \\
(0.00785)\end{array}$ & $\begin{array}{l}-0.0139^{* *} \\
(0.00590)\end{array}$ & $\begin{array}{c}-0.0126 \\
(0.00930)\end{array}$ & $\begin{array}{l}-0.0103^{* * *} \\
(0.00290)\end{array}$ & $\begin{array}{c}-0.0128 \\
(0.00855)\end{array}$ & $\begin{array}{l}-0.00361 \\
(0.00302)\end{array}$ & $\begin{array}{c}-0.0129^{* * *} \\
(0.00370)\end{array}$ & $\begin{array}{c}-0.0467^{* * *} \\
(0.0103)\end{array}$ & $\begin{array}{l}0.00262 \\
(0.0113)\end{array}$ \\
\hline \multicolumn{10}{|l|}{ EDU (base low) } \\
\hline Moderate & $\begin{array}{l}-0.00702 \\
(0.00628)\end{array}$ & $\begin{array}{l}-0.0148^{* * *} \\
(0.00537)\end{array}$ & $\begin{array}{l}-0.0213^{* * *} \\
(0.00723)\end{array}$ & $\begin{array}{l}-0.00923^{* *} \\
(0.00418)\end{array}$ & $\begin{array}{l}-0.00104 \\
(0.00749)\end{array}$ & $\begin{array}{l}-0.0145^{* * *} \\
(0.00373)\end{array}$ & $\begin{array}{l}0.0204^{* * *} \\
(0.00407)\end{array}$ & $\begin{array}{l}0.0149^{* * *} \\
(0.00472)\end{array}$ & $\begin{array}{c}0.00694 \\
(0.00900)\end{array}$ \\
\hline High & $\begin{array}{l}0.000232 \\
(0.00685)\end{array}$ & $\begin{array}{c}-0.0235^{* * *} \\
(0.00534)\end{array}$ & $\begin{array}{l}-0.0285^{* * *} \\
(0.00762)\end{array}$ & $\begin{array}{l}-0.0106^{* *} \\
(0.00416)\end{array}$ & $\begin{array}{l}-0.00654 \\
(0.00679)\end{array}$ & $\begin{array}{c}-0.0282^{* * *} \\
(0.00502)\end{array}$ & $\begin{array}{l}0.0216^{* * *} \\
(0.00397)\end{array}$ & $\begin{array}{l}0.00582^{*} \\
(0.00314)\end{array}$ & $\begin{array}{c}0.0162^{*} \\
(0.00892)\end{array}$ \\
\hline \multicolumn{10}{|l|}{$\begin{array}{l}\text { EMP (base } \\
\text { Employed) }\end{array}$} \\
\hline Others & $\begin{array}{l}-0.0687^{* * *} \\
(0.00932)\end{array}$ & $\begin{array}{l}-0.00293 \\
(0.00743)\end{array}$ & $\begin{array}{l}0.0405^{* * *} \\
(0.00949)\end{array}$ & $\begin{array}{l}0.0189^{* * *} \\
(0.00700)\end{array}$ & $\begin{array}{c}0.0173 \\
(0.0110)\end{array}$ & $\begin{array}{c}0.00332 \\
(0.00293)\end{array}$ & $\begin{array}{l}0.0181^{* * *} \\
(0.00567)\end{array}$ & $\begin{array}{l}0.0118^{* *} \\
(0.00477)\end{array}$ & $\begin{array}{l}-0.0195 \\
(0.0129)\end{array}$ \\
\hline \multicolumn{10}{|l|}{ Sex (base male) } \\
\hline Female & $\begin{array}{c}0.0624^{* * *} \\
(0.0185)\end{array}$ & $\begin{array}{l}0.0316^{* *} \\
(0.0131)\end{array}$ & $\begin{array}{c}-0.0987^{* * *} \\
(0.0165)\end{array}$ & $\begin{array}{c}0.00286 \\
(0.00267)\end{array}$ & $\begin{array}{c}-0.0663^{* * *} \\
(0.0145)\end{array}$ & $\begin{array}{c}0.00418 \\
(0.00403)\end{array}$ & $\begin{array}{l}0.0137^{* *} \\
(0.00547)\end{array}$ & $\begin{array}{l}-0.0150^{* * *} \\
(0.00514)\end{array}$ & $\begin{array}{l}0.0407^{*} \\
(0.0215)\end{array}$ \\
\hline \multicolumn{10}{|l|}{$\begin{array}{l}\text { Marital (base } \\
\text { single) }\end{array}$} \\
\hline Others & $\begin{array}{l}0.0375^{* *} \\
(0.0190)\end{array}$ & $\begin{array}{l}0.0431^{* * *} \\
(0.0118)\end{array}$ & $\begin{array}{c}-0.0933^{* * *} \\
(0.0165)\end{array}$ & $\begin{array}{l}0.00399^{*} \\
(0.00225)\end{array}$ & $\begin{array}{l}-0.0170 \\
(0.0142)\end{array}$ & $\begin{array}{l}0.0190^{* * *} \\
(0.00457)\end{array}$ & $\begin{array}{l}0.0281^{* * *} \\
(0.00692)\end{array}$ & $\begin{array}{l}-0.00555 \\
(0.00512)\end{array}$ & $\begin{array}{c}0.0276 \\
(0.0207)\end{array}$ \\
\hline Children & $\begin{array}{c}-0.00834^{* * *} \\
(0.00161)\end{array}$ & $\begin{array}{l}7.89 \mathrm{e}-05 \\
(0.00124)\end{array}$ & $\begin{array}{l}-0.000538 \\
(0.00200)\end{array}$ & $\begin{array}{c}0.00184 \\
(0.00124)\end{array}$ & $\begin{array}{c}-0.00517^{* *} \\
(0.00202)\end{array}$ & $\begin{array}{c}0.00119 \\
(0.000746)\end{array}$ & $\begin{array}{c}0.00875^{* * *} \\
(0.00143)\end{array}$ & $\begin{array}{c}0.000867 \\
(0.000772)\end{array}$ & $\begin{array}{c}0.00223 \\
(0.00260)\end{array}$ \\
\hline
\end{tabular}




\begin{tabular}{|c|c|c|c|c|c|c|c|c|c|}
\hline Mills & $\begin{array}{l}0.925^{* * *} \\
(0.0818)\end{array}$ & $\begin{array}{l}0.320^{* * *} \\
(0.0279)\end{array}$ & $\begin{array}{l}0.810^{* * *} \\
(0.0397)\end{array}$ & $\begin{array}{l}0.138^{* *} \\
(0.0601)\end{array}$ & $\begin{array}{l}0.603^{* * *} \\
(0.0573)\end{array}$ & $\begin{array}{l}0.288^{* * *} \\
(0.0506)\end{array}$ & $\begin{array}{l}0.195^{* * *} \\
(0.0374)\end{array}$ & $\begin{array}{l}0.342^{* * *} \\
(0.0771)\end{array}$ & $\begin{array}{l}0.229^{* * *} \\
(0.0344)\end{array}$ \\
\hline \multicolumn{10}{|l|}{$\begin{array}{l}\text { Nat (base } \\
\text { Kuwaiti) }\end{array}$} \\
\hline Expat & $\begin{array}{l}0.0484^{* * *} \\
(0.00997)\end{array}$ & $\begin{array}{l}-0.0139^{* *} \\
(0.00645)\end{array}$ & $\begin{array}{l}0.0596^{* * *} \\
(0.00835)\end{array}$ & $\begin{array}{l}0.0248^{* *} \\
(0.0111)\end{array}$ & $\begin{array}{c}0.00290 \\
(0.00838)\end{array}$ & $\begin{array}{l}0.0416^{* * *} \\
(0.00654)\end{array}$ & $\begin{array}{l}0.0258^{* * *} \\
(0.00428)\end{array}$ & $\begin{array}{c}-0.00974^{* * *} \\
(0.00309)\end{array}$ & $\begin{array}{c}-0.00943 \\
(0.0106)\end{array}$ \\
\hline Constant & $\begin{array}{c}-1.106^{* * *} \\
(0.139)\end{array}$ & $\begin{array}{l}0.113^{* * *} \\
(0.0419)\end{array}$ & $\begin{array}{l}-0.0375 \\
(0.0744)\end{array}$ & $\begin{array}{l}-0.418^{*} \\
(0.219)\end{array}$ & $\begin{array}{c}-1.264^{* * *} \\
(0.131)\end{array}$ & $\begin{array}{c}-0.472^{* * *} \\
(0.0981)\end{array}$ & $\begin{array}{c}-0.347^{* * *} \\
(0.0918)\end{array}$ & $\begin{array}{c}-0.790^{* * *} \\
(0.201)\end{array}$ & $\begin{array}{l}0.164^{* * *} \\
(0.0549)\end{array}$ \\
\hline Observations & 1,986 & 2,403 & 2,255 & 1,284 & 2,063 & 2,047 & 1,918 & 1,570 & 2,461 \\
\hline R-squared & 0.417 & 0.414 & 0.583 & 0.322 & 0.257 & 0.464 & 0.303 & 0.380 & 0.046 \\
\hline
\end{tabular}

Robust standard errors in parentheses. ${ }^{* *} \mathrm{p}<0.01,{ }^{* *} \mathrm{p}<0.05,{ }^{*} \mathrm{p}<0.1$.

Appendix 2. Robustness checks for Kuwaiti households

\begin{tabular}{|c|c|c|c|c|c|c|c|c|c|}
\hline VARIABLES & $\begin{array}{c}1) \\
\text { Food }\end{array}$ & $\begin{array}{c}(2) \\
\text { Clothing } \\
\end{array}$ & $\begin{array}{c}(3) \\
\text { Housing } \\
\end{array}$ & $\begin{array}{c}(4) \\
\text { Health }\end{array}$ & $\begin{array}{c}(5) \\
\text { Trans. }\end{array}$ & $\begin{array}{c}(6) \\
\text { Comm. }\end{array}$ & $\begin{array}{l}(7) \\
\text { Rec. }\end{array}$ & $\begin{array}{l}(8) \\
\text { Res. }\end{array}$ & $\begin{array}{l}(9) \\
\text { Misc. }\end{array}$ \\
\hline EXP & $\begin{array}{c}0.0985^{* * *} \\
(0.0165)\end{array}$ & $\begin{array}{c}-0.0283^{* * *} \\
(0.00647)\end{array}$ & $\begin{array}{c}0.0428^{* * *} \\
(0.0124)\end{array}$ & $\begin{array}{l}0.0247^{* * *} \\
(0.00934)\end{array}$ & $\begin{array}{l}0.166^{* * *} \\
(0.0248)\end{array}$ & $\begin{array}{c}0.0439^{* * *} \\
(0.0157)\end{array}$ & $\begin{array}{c}0.0251 \\
(0.0155)\end{array}$ & $\begin{array}{c}0.0436 \\
(0.0272)\end{array}$ & $\begin{array}{l}0.0320^{* * *} \\
(0.00914)\end{array}$ \\
\hline Size & $\begin{array}{l}-0.0243^{*} \\
(0.0148)\end{array}$ & $\begin{array}{c}0.0272^{* * *} \\
(0.0105)\end{array}$ & $\begin{array}{c}-0.0867^{* * *} \\
(0.0156)\end{array}$ & $\begin{array}{l}-0.00263 \\
(0.00216)\end{array}$ & $\begin{array}{c}0.0435^{* * *} \\
(0.0159)\end{array}$ & $\begin{array}{l}-0.00235 \\
(0.00358)\end{array}$ & $\begin{array}{l}-0.0170 \\
(0.0121)\end{array}$ & $\begin{array}{l}-0.0134 \\
(0.0102)\end{array}$ & $\begin{array}{c}0.0204 \\
(0.0184)\end{array}$ \\
\hline Age & $\begin{array}{l}0.00670 \\
(0.0104)\end{array}$ & $\begin{array}{l}-0.0335^{* * *} \\
(0.00896)\end{array}$ & $\begin{array}{c}0.0435^{* * *} \\
(0.0110)\end{array}$ & $\begin{array}{c}-0.00960^{* * *} \\
(0.00273)\end{array}$ & $\begin{array}{l}-0.0195 \\
(0.0138)\end{array}$ & $\begin{array}{l}-0.0176^{* * *} \\
(0.00478)\end{array}$ & $\begin{array}{l}-0.00715 \\
(0.00439)\end{array}$ & $\begin{array}{l}-0.0149^{*} \\
(0.00805)\end{array}$ & $\begin{array}{l}-0.00968 \\
(0.0155)\end{array}$ \\
\hline \multicolumn{10}{|l|}{$\begin{array}{c}\text { EDU (base } \\
\text { low) }\end{array}$} \\
\hline Moderate & $\begin{array}{c}-0.0113 \\
(0.00877)\end{array}$ & $\begin{array}{c}-0.0310^{* * *} \\
(0.00977)\end{array}$ & $\begin{array}{c}0.00469 \\
(0.00979)\end{array}$ & $\begin{array}{c}-0.00461^{* *} \\
(0.00200)\end{array}$ & $\begin{array}{c}-0.0331^{* *} \\
(0.0129)\end{array}$ & $\begin{array}{c}-0.00768^{* *} \\
(0.00301)\end{array}$ & $\begin{array}{c}0.0189^{* *} \\
(0.00858)\end{array}$ & $\begin{array}{c}-0.0150 \\
(0.00926)\end{array}$ & $\begin{array}{l}0.00789 \\
(0.0141)\end{array}$ \\
\hline High & $\begin{array}{l}-0.0206^{*} \\
(0.0120)\end{array}$ & $\begin{array}{c}-0.0568^{* * *} \\
(0.0110)\end{array}$ & $\begin{array}{c}0.0323^{* * *} \\
(0.0124)\end{array}$ & $\begin{array}{l}-0.0144^{* * *} \\
(0.00393)\end{array}$ & $\begin{array}{c}-0.0329^{* *} \\
(0.0138)\end{array}$ & $\begin{array}{c}-0.0288^{* * *} \\
(0.0101)\end{array}$ & $\begin{array}{l}-0.0151^{*} \\
(0.00782)\end{array}$ & $\begin{array}{l}-0.0176 \\
(0.0141)\end{array}$ & $\begin{array}{c}0.0156 \\
(0.0168)\end{array}$ \\
\hline \multicolumn{10}{|l|}{ EMP (base } \\
\hline Others & $\begin{array}{c}-0.0482^{* * *} \\
(0.0108)\end{array}$ & $\begin{array}{l}-0.00230 \\
(0.00940)\end{array}$ & $\begin{array}{c}0.0381^{* * *} \\
(0.0113)\end{array}$ & $\begin{array}{l}0.0158^{* * *} \\
(0.00468)\end{array}$ & $\begin{array}{c}0.0194 \\
(0.0151)\end{array}$ & $\begin{array}{l}0.0163^{* * *} \\
(0.00374)\end{array}$ & $\begin{array}{c}0.00805 \\
(0.00721)\end{array}$ & $\begin{array}{c}0.00376 \\
(0.00477)\end{array}$ & $\begin{array}{l}-0.0171 \\
(0.0159)\end{array}$ \\
\hline $\begin{array}{l}\text { Sex (base } \\
\text { male) }\end{array}$ & & & & & & & & & \\
\hline Female & $\begin{array}{l}-0.00902 \\
(0.0250)\end{array}$ & $\begin{array}{l}0.0334^{*} \\
(0.0185)\end{array}$ & $\begin{array}{l}-0.0128 \\
(0.0196)\end{array}$ & $\begin{array}{c}0.00381 \\
(0.00290)\end{array}$ & $\begin{array}{l}-0.109^{* * *} \\
(0.0234)\end{array}$ & $\begin{array}{c}0.00454 \\
(0.00563)\end{array}$ & $\begin{array}{c}0.0104 \\
(0.00735)\end{array}$ & $\begin{array}{l}-0.00374 \\
(0.00524)\end{array}$ & $\begin{array}{l}0.00663 \\
(0.0276)\end{array}$ \\
\hline \multicolumn{10}{|l|}{$\begin{array}{l}\text { Marital (base } \\
\text { single) }\end{array}$} \\
\hline Others & $\begin{array}{l}0.00676 \\
(0.0246)\end{array}$ & $\begin{array}{c}0.0604^{* * *} \\
(0.0172)\end{array}$ & $\begin{array}{l}0.00494 \\
(0.0181)\end{array}$ & $\begin{array}{l}0.000936 \\
(0.00246)\end{array}$ & $\begin{array}{l}-0.0402^{*} \\
(0.0224)\end{array}$ & $\begin{array}{c}0.0121^{*} \\
(0.00676)\end{array}$ & $\begin{array}{l}0.0376^{* *} \\
(0.0163)\end{array}$ & $\begin{array}{c}0.0151 \\
(0.0110)\end{array}$ & $\begin{array}{l}-0.0295 \\
(0.0272)\end{array}$ \\
\hline Children & $\begin{array}{c}-0.00537^{* *} \\
(0.00264)\end{array}$ & $\begin{array}{c}-0.00543^{* * *} \\
(0.00208)\end{array}$ & $\begin{array}{l}0.0160^{* * *} \\
(0.00312)\end{array}$ & $\begin{array}{l}0.00195^{* * *} \\
(0.000697)\end{array}$ & $\begin{array}{c}-0.00730^{* *} \\
(0.00317)\end{array}$ & $\begin{array}{l}0.00312^{* * *} \\
(0.000998)\end{array}$ & $\begin{array}{l}0.00826^{* *} \\
(0.00338)\end{array}$ & $\begin{array}{l}0.00555^{*} \\
(0.00308)\end{array}$ & $\begin{array}{c}-0.00414 \\
(0.00386)\end{array}$ \\
\hline Mills & $\begin{array}{c}0.827^{* * *} \\
(0.102)\end{array}$ & $\begin{array}{l}0.312^{* * *} \\
(0.0313)\end{array}$ & $\begin{array}{l}0.849^{* * *} \\
(0.0641)\end{array}$ & $\begin{array}{l}0.109^{* * *} \\
(0.0315)\end{array}$ & $\begin{array}{c}0.756^{* * *} \\
(0.113)\end{array}$ & $\begin{array}{l}0.264^{* * *} \\
(0.0824)\end{array}$ & $\begin{array}{l}0.200^{* *} \\
(0.0849)\end{array}$ & $\begin{array}{l}0.212^{*} \\
(0.115)\end{array}$ & $\begin{array}{l}0.341^{* * *} \\
(0.0661)\end{array}$ \\
\hline Constant & $\begin{array}{c}-0.965^{* * *} \\
(0.175)\end{array}$ & $\begin{array}{l}0.284^{* * *} \\
(0.0578)\end{array}$ & $\begin{array}{c}-0.274^{* *} \\
(0.113)\end{array}$ & $\begin{array}{c}-0.269^{* * *} \\
(0.101)\end{array}$ & $\begin{array}{c}-1.501^{* * *} \\
(0.237)\end{array}$ & $\begin{array}{c}-0.436^{* * *} \\
(0.159)\end{array}$ & $\begin{array}{l}-0.271^{*} \\
(0.164)\end{array}$ & $\begin{array}{l}-0.430 \\
(0.275)\end{array}$ & $\begin{array}{c}0.187^{* *} \\
(0.0824)\end{array}$ \\
\hline Observations & 795 & 983 & 910 & 541 & 857 & 806 & 784 & 690 & 1,016 \\
\hline R-squared & 0.381 & 0.407 & 0.577 & 0.417 & 0.210 & 0.456 & 0.257 & 0.261 & 0.056 \\
\hline
\end{tabular}

Robust standard errors in parentheses. ${ }^{* * *} \mathrm{p}<0.01,{ }^{* *} \mathrm{p}<0.05,{ }^{*} \mathrm{p}<0.1$. 
Appendix 3. Robustness checks for Expatriates households

\begin{tabular}{|c|c|c|c|c|c|c|c|c|c|}
\hline VARIABLES & $\begin{array}{c}(1) \\
\text { Food }\end{array}$ & $\begin{array}{c}(2) \\
\text { Clothing } \\
\end{array}$ & $\begin{array}{c}(3) \\
\text { Housing } \\
\end{array}$ & $\begin{array}{c}(4) \\
\text { Health }\end{array}$ & $\begin{array}{c}(5) \\
\text { Trans. }\end{array}$ & $\begin{array}{c}(6) \\
\text { Comm. }\end{array}$ & $\begin{array}{l}(7) \\
\text { Rec. }\end{array}$ & $\begin{array}{r}(8) \\
\text { Res. }\end{array}$ & $\begin{array}{c}(9) \\
\text { Misc. }\end{array}$ \\
\hline EXP & $\begin{array}{c}0.0854^{* * *} \\
(0.0176)\end{array}$ & $\begin{array}{c}0.00878 \\
(0.00539)\end{array}$ & $\begin{array}{l}0.00955 \\
(0.0115)\end{array}$ & $\begin{array}{c}0.0628 \\
(0.0391)\end{array}$ & $\begin{array}{l}0.126^{* * *} \\
(0.0154)\end{array}$ & $\begin{array}{c}0.0572^{* * *} \\
(0.0127)\end{array}$ & $\begin{array}{l}0.0222^{* * *} \\
(0.00713)\end{array}$ & $\begin{array}{l}0.137^{* * *} \\
(0.0335)\end{array}$ & $\begin{array}{l}0.0358^{* * *} \\
(0.00851)\end{array}$ \\
\hline Size & $\begin{array}{l}0.115^{* * *} \\
(0.0163)\end{array}$ & $\begin{array}{l}-0.00474 \\
(0.00897)\end{array}$ & $\begin{array}{c}-0.0544^{* * *} \\
(0.0148)\end{array}$ & $\begin{array}{l}-0.0192^{* *} \\
(0.00929)\end{array}$ & $\begin{array}{c}0.0320^{* * *} \\
(0.0107)\end{array}$ & $\begin{array}{l}-0.0210^{* * *} \\
(0.00757)\end{array}$ & $\begin{array}{l}-0.00336 \\
(0.00513)\end{array}$ & $\begin{array}{l}0.0166^{* * *} \\
(0.00600)\end{array}$ & $\begin{array}{l}-0.00646 \\
(0.0170)\end{array}$ \\
\hline Age & $\begin{array}{c}0.0145^{* *} \\
(0.00599)\end{array}$ & $\begin{array}{l}1.88 \mathrm{e}-05 \\
(0.00440)\end{array}$ & $\begin{array}{c}-0.0208^{* * *} \\
(0.00740)\end{array}$ & $\begin{array}{c}-0.00659^{* *} \\
(0.00279)\end{array}$ & $\begin{array}{c}-0.0217^{* * *} \\
(0.00573)\end{array}$ & $\begin{array}{l}-0.00186 \\
(0.00235)\end{array}$ & $\begin{array}{l}-0.000525 \\
(0.00297)\end{array}$ & $\begin{array}{c}-0.0723^{* * *} \\
(0.0167)\end{array}$ & $\begin{array}{l}-0.00247 \\
(0.00849)\end{array}$ \\
\hline $\begin{array}{c}\text { EDU (base } \\
\text { low) }\end{array}$ & & & & & & & & & \\
\hline Moderate & $\begin{array}{c}0.00169 \\
(0.00888)\end{array}$ & $\begin{array}{l}-0.00858 \\
(0.00587)\end{array}$ & $\begin{array}{c}-0.0463^{* * *} \\
(0.0106)\end{array}$ & $\begin{array}{l}-0.0139^{*} \\
(0.00779)\end{array}$ & $\begin{array}{l}0.0173^{* *} \\
(0.00840)\end{array}$ & $\begin{array}{c}-0.0177^{* * *} \\
(0.00511)\end{array}$ & $\begin{array}{l}0.0219^{* * *} \\
(0.00381)\end{array}$ & $\begin{array}{c}0.0726^{* * *} \\
(0.0187)\end{array}$ & $\begin{array}{c}0.0133 \\
(0.0118)\end{array}$ \\
\hline High & $\begin{array}{l}0.0278^{* * *} \\
(0.00854)\end{array}$ & $\begin{array}{l}-0.00464 \\
(0.00535)\end{array}$ & $\begin{array}{c}-0.0742^{* * * *} \\
(0.0100)\end{array}$ & $\begin{array}{c}-0.00539^{* *} \\
(0.00260)\end{array}$ & $\begin{array}{c}0.00188 \\
(0.00695)\end{array}$ & $\begin{array}{l}-0.0269^{* * *} \\
(0.00552)\end{array}$ & $\begin{array}{l}0.0384^{* * *} \\
(0.00546)\end{array}$ & $\begin{array}{c}0.0514^{* * *} \\
(0.0135)\end{array}$ & $\begin{array}{l}0.0223^{* *} \\
(0.0106)\end{array}$ \\
\hline \multicolumn{10}{|l|}{$\begin{array}{l}\text { EMP (base } \\
\text { Employed) }\end{array}$} \\
\hline Others & $\begin{array}{c}-0.0684^{* * *} \\
(0.0210)\end{array}$ & $\begin{array}{c}0.0286 \\
(0.0177)\end{array}$ & $\begin{array}{l}0.0530^{* *} \\
(0.0254)\end{array}$ & $\begin{array}{l}0.0489^{* *} \\
(0.0241)\end{array}$ & $\begin{array}{l}0.00215 \\
(0.0274)\end{array}$ & $\begin{array}{l}-0.0139^{* *} \\
(0.00707)\end{array}$ & $\begin{array}{l}-0.00662 \\
(0.00813)\end{array}$ & $\begin{array}{c}-0.0351^{* * *} \\
(0.0112)\end{array}$ & $\begin{array}{l}-0.0362 \\
(0.0336)\end{array}$ \\
\hline \multicolumn{10}{|l|}{$\begin{array}{c}\text { Sex (base } \\
\text { male) }\end{array}$} \\
\hline Female & $\begin{array}{l}0.167^{* * *} \\
(0.0322)\end{array}$ & $\begin{array}{c}0.0229 \\
(0.0149)\end{array}$ & $\begin{array}{l}-0.184^{* * *} \\
(0.0300)\end{array}$ & $\begin{array}{l}-0.00533 \\
(0.00379)\end{array}$ & $\begin{array}{c}-0.00308 \\
(0.0165)\end{array}$ & $\begin{array}{c}0.00482 \\
(0.00685)\end{array}$ & $\begin{array}{c}0.0171^{* *} \\
(0.00720)\end{array}$ & $\begin{array}{l}-0.0206^{* *} \\
(0.00934)\end{array}$ & $\begin{array}{l}0.0893^{* *} \\
(0.0355)\end{array}$ \\
\hline \multicolumn{10}{|l|}{$\begin{array}{l}\text { Marital (base } \\
\text { single) }\end{array}$} \\
\hline Others & $\begin{array}{c}0.0761^{* * *} \\
(0.0275)\end{array}$ & $\begin{array}{l}0.0281^{*} \\
(0.0144)\end{array}$ & $\begin{array}{l}-0.199^{* * *} \\
(0.0300)\end{array}$ & $\begin{array}{l}0.00616^{*} \\
(0.00330)\end{array}$ & $\begin{array}{r}-0.00340 \\
(0.0159)\end{array}$ & $\begin{array}{l}0.0111^{* *} \\
(0.00503)\end{array}$ & $\begin{array}{l}0.0208^{* * *} \\
(0.00721)\end{array}$ & $\begin{array}{c}-0.0578^{* * *} \\
(0.0149)\end{array}$ & $\begin{array}{l}0.0789^{* *} \\
(0.0306)\end{array}$ \\
\hline Children & $\begin{array}{c}-0.0319^{* * *} \\
(0.00471)\end{array}$ & $\begin{array}{c}0.00774^{* * *} \\
(0.00246)\end{array}$ & $\begin{array}{l}-0.00136 \\
(0.00448)\end{array}$ & $\begin{array}{c}0.000317 \\
(0.000940)\end{array}$ & $\begin{array}{c}-0.0118^{* * *} \\
(0.00338)\end{array}$ & $\begin{array}{c}-0.00427^{* * *} \\
(0.00155)\end{array}$ & $\begin{array}{l}0.0113^{* * *} \\
(0.00191)\end{array}$ & $\begin{array}{l}-0.0137^{* * *} \\
(0.00347)\end{array}$ & $\begin{array}{l}-0.00371 \\
(0.00485)\end{array}$ \\
\hline Mills & $\begin{array}{c}0.933^{* * *} \\
(0.122)\end{array}$ & $\begin{array}{l}0.334^{* * *} \\
(0.0387)\end{array}$ & $\begin{array}{l}0.743^{* * *} \\
(0.0538)\end{array}$ & $\begin{array}{c}0.176^{*} \\
(0.0969)\end{array}$ & $\begin{array}{l}0.497^{* * *} \\
(0.0613)\end{array}$ & $\begin{array}{l}0.311^{* * *} \\
(0.0599)\end{array}$ & $\begin{array}{l}0.163^{* * *} \\
(0.0279)\end{array}$ & $\begin{array}{c}0.536^{* * *} \\
(0.124)\end{array}$ & $\begin{array}{l}0.165^{* * *} \\
(0.0433)\end{array}$ \\
\hline Constant & $\begin{array}{c}-1.131^{* * *} \\
(0.207)\end{array}$ & $\begin{array}{l}-0.0399 \\
(0.0489)\end{array}$ & $\begin{array}{l}0.354^{* * *} \\
(0.0971)\end{array}$ & $\begin{array}{l}-0.588 \\
(0.374)\end{array}$ & $\begin{array}{c}-1.117^{* * *} \\
(0.142)\end{array}$ & $\begin{array}{c}-0.465^{* * *} \\
(0.108)\end{array}$ & $\begin{array}{c}-0.247^{* * *} \\
(0.0700)\end{array}$ & $\begin{array}{c}-1.355^{* * *} \\
(0.337)\end{array}$ & $\begin{array}{c}0.104 \\
(0.0773)\end{array}$ \\
\hline Observations & 1,191 & 1,420 & 1,345 & 743 & 1,206 & 1,241 & 1,134 & 880 & 1,445 \\
\hline R-squared & 0.448 & 0.436 & 0.577 & 0.348 & 0.261 & 0.506 & 0.309 & 0.464 & 0.032 \\
\hline
\end{tabular}

Robust standard errors in parentheses. ${ }^{* * *} \mathrm{p}<0.01,{ }^{* *} \mathrm{p}<0.05,{ }^{*} \mathrm{p}<0.1$.

\section{References}

Alomar, A. S. A. E. (2015). Leadership effectiveness from the perspective of chief executive officers in Kuwait. The University of Bradford. Retrieved https://bradscholars.brad.ac.uk/bitstream/handle/10454/11881/UB_09020473\%20Abeer\%20AlomarResubmitting\%20jan.pdf?sequence $=1 \&$ isAllowed $=\mathrm{y}$

Barnett, W. A., \& Serletis, A. (2008). Measuring Consumer Preferences and Estimating Demand Systems (No. 12318). University Library of Munich, Germany.

Burney, N. A., and Al-Mutairi, N. (1993). Household consumption patterns in Kuwait. The Middle Eastern Business and Economic Review, 5(2), 1-12.

Bushway, S., Johnson, B. D. and Slocum, L. A. (2007). Is the magic still there? The use of the Heckman two-step correction for selection bias in criminology. Journal of Quantitative Criminology, 23(2), 151-178.

Central Bank of Kuwait (CBK). (2016). Quarterly Statistical Bulletin (Special Edition). Retrieved from apahttp://www.cbk.gov.kw/WWW/index.html

Chandran, V. V., Geetha, C., Yii, K. J. and Ahmed, A. (2015). An empirical analysis of the Engel curve on energy for households in Sabah and Sarawak based on location and income group. The Journal of Developing Areas, 49(5), 239-25.

De Agostini, P. (2014). The effect of food prices and household income on the British diet (No. 2014-10). ISER Working Paper Series. 
Deaton, A. and Muellbauer, J. (1980). Economics and consumer behaviour. Cambridge, UK: Cambridge University Press.

Durham, C. and Eales, J. (2010). Demand elasticities for fresh fruit at the retail level. Applied Economics, 42(11), pp.1345-1354.

Dybczak, K., Tóth, P. and Voňka, D. (2014). Effects of price shocks on consumer demand: Estimating the QUADS demand system on Czech household budget survey data. Czech Journal of Economics and Finance, 64(6), 476500.

Heckman, J. J. (1979). Sample selection bias as a specification error. Econometrica: Journal of the econometric society, 153-161.

Heien, D. and Wessells, C. R. (1990). Demand systems estimation with microdata: A censored regression approach. Journal of Business E Economic Statistics, 8(3), 365-371.

Houthakker, H. S. (1957). An international comparison of household expenditure patterns, commemorating the centenary of Engel's law. Econometrica, Journal of the Econometric Society, 532-551.

Jabarin, A. S. (2005). Estimation of meat demand system in Jordan: an almost ideal demand system. International Journal of consumer studies, 29(3), 232-238.

Kaabia, M. B., \& Gil, J. M. (2001). Estimation and inference in cointegrated demand systems: an application to Tunisian meat consumption. European Review of Agricultural Economics, 28(3), 349-370.

Leser, C. E. V. (1963). Forms of Engel functions. Econometrica: Journal of the Econometric Society, 694-703.

Michelini, C., \& Chatterjee, S. (1997). Demographic variables in demand systems: An analysis of New Zealand household expenditure 1984-1992. New Zealand Economic Papers, 31(2), 153-173.

Mok, T. P., Maclean, G., \& Dalziel, P. (2011). Household size economies: Malaysian evidence. Economic Analysis $\mathcal{E}$ Policy, 41(2), 203-223.

Setterfield, M., \& Kim, Y. K. (2017). Household borrowing and the possibility of 'consumption-driven, profit-led growth'. Review of Keynesian Economics, 5(1), 43-60.

Tian, X., Geng, Y., Dai, H., Fujita, T., Wu, R., Liu, Z., ... \& Yang, X. (2016). The effects of household consumption pattern on regional development: a case study of Shanghai. Energy, 103, 49-60.

Working, H. (1943). Statistical laws of family expenditure. Journal of the American Statistical Association, 38(221), 4356.

Yeong-Sheng, T. E. Y., Shamsudin, M. N., Mohamed, Z., \& Mahir Abdullah, A. (2009). Demand analyses of rice in Malaysia. MPRA Paper, (15062).

Yusof, S. A., \& Duasa, J. (2010). Household decision-making and expenditure patterns of married men and women in Malaysia. Journal of family and economic issues, 31(3), 371-381.

Zhang, X., \& Goddard, E. (2010). Analysis of value-added meat product choice behaviour by Canadian households. Rural Economy Project Report-Department of Rural Economy, University of Alberta, (10-04)

(C) 2020 by the authors. This article is an open-access article distributed under the terms and conditions of the Creative Commons Attribution (CC BY) license (http://creativecommons.org/licenses/by/4.0/). 